Higher dimensional complex geometry

Eduard Looijenga 



\section{Cohomology and intersection homology of algebraic varieties Eduard Looijenga}

\section{Introduction}

These is the write up of a series of lectures that intended to give an overview of the algebraic topology of complex algebraic varieties. The most important tool here is the theory of Lefschetz pencils, which serves in algebraic geometry similar purposes as Morse theory in differential topology.

The first four lectures are devoted to the classical aspects of this theory: Lecture 1 deals with dual varieties and it shown there that there are plenty Lefschetz pencils on a smooth projective variety with good properties. Lecture 2 is devoted to the weak Lefschetz theorem, lecture 3 discusses various properties that are equivalent to the hard Lefschetz theorem and lecture 4 is about monodromy and the PicardLefschetz formula's. Here I followed to a large extent Lamotke's survey [12].

The standard proof of the hard Lefschetz theorem is due to Hodge and is based his theory of harmonic forms. Until the mid-seventies this was the only one. When Deligne proved in $[7]$ the corresponding result for varieties defined over finite fields, he obtained at the same time another proof in the complex case (because there is a comparison theorem and a base change property). That proof made ingeneous use of Lefschetz pencils. Ideas of that proof have been used to obtain far reaching generalizations.

One of these generalizations concerns singular projective varieties. It was clear from the outset that the Lefschetz theorems could not hold for such varieties, simply because of the failure of Poincare duality. However, with the discovery of intersection homology by Goresky-MacPherson, a homology theory for singular spaces had arrived that had Poincaré duality built in. Shortly afterwards, Bernstein, Beilinson, Deligne and Gabber showed that singular projective varieties defined over finite fields satisfy the hard Lefschetz theorem if we replace étale cohomology by the intersection homology analog. They also proved relative versions of this result,

\footnotetext{
${ }^{1}$ Faculteit Wiskunde en Informatica, Rijksuniversiteit Utrecht, PO Box 80.010, 3508 TA Utrecht, The Netherlands

E-mail address: looijenga@math.ruu.nl 
among which the decomposition theorem. This led M. Saito to develop the complex (or rather Kähler) counterpart of these results.

We have attempted to describe some of the ideas that go into this generalization in the last two sections. Lecture 5 is devoted to a detailed study of the Leray spectral sequence of a Lefschetz pencil following Grothendieck and Deligne. We use this to outline a proof of the hard Lefschetz theorem for smooth varieties of which the analytic input is given by Zucker's fundamental theorem on the cohomology of curves with values in polarized Hodge structure with degeneracies. This proof is admittedly more involved than the standard proof, but has the virtue that it generalizes to intersection homology of singular projective varieties. That generalization is the topic of lecture 6 .

These lectures were based on a (part of a) course I gave at the University of Utah in the Spring of 1991. During that course, Herb Clemens carefully took notes. These notes were available to me (and the audience) while I gave the lectures and proved very useful in writing this up. I thank him heartily.

A word of warning regarding the bibliography is perhaps in order: this is merely a list of works that we referred to and there is no pretense of completeness of any kind. But we do hope that it may help the reader to find further references. 


\section{LECTURE 1. The dual variety and Lefschetz pencils}

Throughout this lecture we fix a complex vector space $V$ of dimension $N+1$. We denote by $\mathbb{P}:=\mathbb{P}(V)$ be its associated projective space (its points parametrize the one-dimensional subspaces of $V$ ) and by $\mathbb{P}^{*}:=\mathbb{P}\left(V^{*}\right)$ the dual (whose points parametrize the linear hyperplanes of $V$, or equivalently, the projective hyperplanes of $\mathbb{P})$. We write $H_{\xi} \subset \mathbb{P}$ for the projective hyperplane labeled by $\xi \in \mathbb{P}^{*}$. Likewise, $x \in \mathbb{P}$ determines a hyperplane $H_{x}$ of $\mathbb{P}^{*}$.

The pairs $(x, \xi) \in \mathbb{P} \times \mathbb{P}^{*}$ with the property that $x \in H_{\xi}$ (or equivalently, $\left.\xi \in H_{x}\right)$ make up a closed subvariety $W$ of $\mathbb{P} \times \mathbb{P}^{*}$, called the incidence variety. A choice of a basis for $V$ determines projective coordinates $\left[x^{0}: \ldots x^{N}\right]$ for $\mathbb{P}$ and $\left[\xi_{0}: \ldots \xi_{N}\right]$ for $\mathbb{P}^{*}$; in terms of these coordinates $W$ is given by the single equation $\sum_{i=0}^{N} x^{i} \xi_{i}=0$. It is a smooth hypersurface in $\mathbb{P} \times \mathbb{P}^{*}$. Let

$$
p: W \rightarrow \mathbb{P} \quad, \quad q: W \rightarrow \mathbb{P}^{*}
$$

be the two projections.

(1.1) Lemma. The projections $p$ and $q$ are locally trivial with fibre a projective space of dimension $N-1$.

The proof is left to you.

Now let $X \subset \mathbb{P}$ be closed irreducible subvariety of dimension $n<N$. If $x$ is a smooth point of $X$, then let $\mathbb{T}_{x} X$ denote the projective $n$-dimensional subspace of $\mathbb{P}$ spanned by the tangent space of $X$ at $x$. It is clear that $H_{\xi}$ is tangent to $X$ at $x$ iff $H_{\xi} \supset \mathbb{T}_{x} X$. We define the projectivized conormal bundle of the smooth part $X_{\text {reg }}$ of $X$ by

$$
N_{X_{\text {reg }}}:=\left\{(x, \xi) \in X_{\text {reg }} \times \mathbb{P}^{*}: H_{\xi} \supset \mathbb{T}_{x} X\right\} .
$$

(1.2) Lemma. $N_{X_{\mathrm{reg}}}$ is a closed irreducible subvariety of $X_{\mathrm{reg}} \times \mathbb{P}^{*}$ of dimension $N-1$ and the projection $N_{X_{\mathrm{reg}}} \rightarrow X_{\mathrm{reg}}$ is locally trivial with fibre a projective space of dimension $N-n-1$.

Proof. Given $x \in X_{\text {reg }}$, choose a complementary projective subspace $K$ to $\mathbb{T}_{x} X$ in $\mathbb{P}$. Then the set of $y \in X_{\text {reg }}$ with $\mathbb{T}_{y} X \cap K=\emptyset$ is a Zariski open neighborhood 
$U$ of $x$ and we have a natural isomorphism $p^{-1}(U) \cap N_{X_{\text {reg }}} \cong U \times K^{*}$. This is a local trivialization.

We write $N_{X}$ for the Zariski closure of $N_{X_{\text {reg }}}$ in $W$. This is a subvariety of dimension $N-1$. Clearly, $p\left(N_{X}\right)=X$. The image $q\left(N_{X}\right)$ is called the dual variety of $X$; it is usually denoted by $X^{\vee}$. It is a closed irreducible subvariety of $\mathbb{P}^{*}$.

(1.3) Proposition. The exchange $\tau: \mathbb{P} \times \mathbb{P}^{*} \cong \mathbb{P}^{*} \times \mathbb{P}$ maps $N_{X}$ onto $N_{X^{\vee}}$ and so $X^{\vee \vee}=X$.

We put $W_{X}:=p^{-1}(X) \cap W$ and $q_{X}:=q \mid W_{X}$. Since $p: W_{X} \rightarrow X$ is locally trivial with smooth fibre, $W_{X, \mathrm{reg}}=W_{X_{\mathrm{reg}}}$. We first prove:

(1.4) Lemma. For $(a, \alpha) \in W_{X \text {,reg, the following are equivalent: }}$

1. $q_{X}$ has not the maximal rank $N$ at $(a, \alpha)$,

2. $(a, \alpha) \in N_{X}$, i.e., $H_{\alpha} \supset \mathbb{T}_{a} X$,

3. $d q\left(T_{(a, \alpha)} W_{X}\right)=T_{\alpha} H_{a}$.

Proof. Choose a basis for $V$ such that $a=[1: 0: \cdots: 0]$ and $H_{\alpha}$ is given by $x^{N}=0$. Then in terms of the obvious affine coordinates $q_{X}$ is given by

$$
\left(x ; u_{1}, \ldots, u_{N-1}\right) \mapsto\left(-\sum_{i=1}^{N-1} x^{i} u_{i}-x^{N}, u_{1}, \ldots, u_{N-1}\right),
$$

where $x=\left[1: x^{1}: \cdots: x^{N}\right]$. It is clear that $q_{X}$ fails to have rank $N$ at $(a, \alpha)$ iff $x_{N} \mid X$ has $a$ as a critical point. This last property just means that $H_{\alpha} \supset \mathbb{T}_{a} X$. This proves $(1) \Leftrightarrow(2)$.

Since $W_{X}$ contains $\{a\} \times H_{a}$, we have

$$
T_{\alpha} H_{a} \subset d q\left(T_{(a, \alpha)} W_{X}\right) .
$$

The left-hand side has dimension $N-1$. So if the right-hand side has dimension at most $N-1$, this inclusion must be an equality. This proves $(1) \Leftrightarrow(3)$.

Proof of (1.3). Let $U$ be an open-dense subset of $N_{X_{\text {reg }}}$ with the property for all $(a, \alpha) \in U, \alpha \in X_{\text {reg }}^{\vee}$ and $d q\left(T_{(a, \alpha)} N_{X}\right)=T_{\alpha} X^{\vee}$. By lemma (1.3) we have

$$
T_{\alpha} H_{a}=d q\left(T_{(a, \alpha)} W_{X}\right)
$$

for all $(a, \alpha) \in N_{X_{\text {reg }}}$. So if $(a, \alpha) \in U$, then $T_{\alpha} H_{a} \supset T_{\alpha} X^{\vee}$. This means that $(\alpha, a) \in N_{X^{\vee}}$. We have proved that $\tau(U) \subset N_{X^{\vee}}$. Since both $N_{X}$ and $N_{X^{\vee}}$ are closed and irreducible of dimension $N-1$, it follows that $\tau\left(N_{X}\right)=N_{X}$.

(1.5) Corollary. If $X$ is smooth, then the set of critical points of $q_{X}$ is equal to $N_{X}$ and hence its set of critical values is $X^{\vee}$. In particular, $q_{X}$ is locally trivial in the $C^{\infty}$ sense over $\mathbb{P}^{*}-X^{\vee}$.

Proof. The first statement is immediate from lemma (1.4). The last clause follows from the Ehresmann fibration theorem. 
(1.6) Corollary. All smooth hypersurfaces in $\mathbb{P}^{n}$ of a fixed degree $d>0$ are mutually diffeomorphic.

Proof. Apply the proposition to the image $X$ of the $d$-fold Veronese embedding $f: \mathbb{P}^{n} \rightarrow \mathbb{P}^{N}$ (where $N+1$ is the number of monomials of degree $d$ in $n+1$ variables, and $f$ has these monomials as coordinates).

In the remainder of this lecture we assume that $X$ is smooth.

Let $L$ be line in $\mathbb{P}^{*}$. It determines a codimension two linear subspace $A$ of $\mathbb{P}$ with the property that

$$
\xi \in L \Leftrightarrow A \subset H_{\xi}
$$

The family of hyperplanes of $\mathbb{P}$ parametrized by $L$ is called a pencil. We put

$$
\tilde{X}:=q_{X}^{-1}(L)=\left\{(x, \xi) \in X \times L: x \in H_{\xi}\right\}
$$

and we denote the two by projections by $\pi: \tilde{X} \rightarrow X$ and $f: \tilde{X} \rightarrow X$. We further put $Y:=X \cap A$. Observe that then

- $\pi^{-1} Y=Y \times L$; we shall often write $\tilde{Y}$ for this space,

- $\pi: \tilde{X}-\tilde{Y} \rightarrow X-Y$ is an isomorphism, the inverse mapping being given by $x \mapsto(x, \operatorname{span}\langle x, A\rangle)$,

- $f^{-1}(\xi)=H_{\xi} \cap X$; this intersection will also be denoted by $X_{\xi}$.

We say that $L$ is transversal to $X^{\vee}$ if $L$ meets $X^{\vee}$ in its smooth part only and is transversal to $X_{\text {reg }}^{\vee}$. We first show that such lines are plenty :

(1.7) Lemma. Given $\alpha \in \mathbb{P}^{*}-X^{\vee}$, then the lines in $\mathbb{P}^{*}$ through $\alpha$ form a projective space $\mathbb{P}_{\alpha}$ of dimension $N-1$ of which an open dense subset parametrizes $X^{\vee}$-transversal lines.

Proof. Let $\phi: X^{\vee} \rightarrow \mathbb{P}_{\alpha}$ be the map which assigns to $\xi \in X^{\vee}$ the line spanned by $\xi$ and $\alpha$. Then $\xi \in X_{\text {reg }}^{\vee}$ is a critical point of $\phi$ iff $\operatorname{span}(\xi, \alpha)$ is contained in $\mathbb{T}_{\xi} X^{\vee}$. So the set of lines passing through $\alpha$ which are not transversal to $X^{\vee}$ is the union of $\phi\left(X_{\text {sing }}\right)$ and the set of critical values of $\phi \mid X_{\text {reg }}^{\vee}$, and hence is contained in a proper subvariety.

The number of points of intersection of an $X^{\vee}$-transversal line with $X^{\vee}$ is independent of $L$; this number is called the class of $X$. If $X^{\vee}$ is a hypersurface, then the class of $X$ is simply the degree of $X^{\vee}$; else it is zero.

Let us fix an $X^{\vee}$-transversal line $L$.

(1.8) Proposition. The following properties hold:

1. A is transversal to $X$, so that $Y$ is smooth in $X$ of codimension two,

2. $\tilde{X}$ is smooth,

3. no critical point of $f$ lies in $\tilde{Y}$ and $f$ maps its critical points bijectively onto $L \cap X^{\vee}$,

4. each critical point $\tilde{x}$ of $f$ is nondegenerate, i.e., the hessian of $f$ at such a point (which is a bilinear map $T_{\tilde{x}} \tilde{X} \times T_{\tilde{x}} \tilde{X} \rightarrow T_{f(\tilde{x})} L$ ) is nondegenerate. 
Proof. (1) Assume $A$ is not transversal to $X$ at $x$. Then there is a hyperplane $H$ containing $\mathbb{T}_{x} X \cup A$. So $H=H_{\xi}$ for some $\xi \in \mathrm{L}$ and $(x, \xi) \in N_{X}$. Hence $\xi \in V^{\vee}$. But then $\xi \in X_{\text {reg }}^{\vee}$ since $L$ is transversal to $X^{\vee}$. Since $(\xi, x) \in N_{X^{\vee}}$, it follows that $\mathbb{T}_{\xi} X^{\vee} \subset H_{x}$. Since $x \in A$, we also have $L \subset H_{x}$. So $\mathbb{T}_{\xi} X^{\vee}+L \subset H_{x}$, which contradicts the fact that $L$ is transversal to $X^{\vee}$.

(2) Since $\tilde{X}=q_{X}^{-1}(L)$, it is enough to show that $q: W_{X} \rightarrow \mathbb{P}^{*}$ is tranversal to $L$. Let $(a, \alpha) \in \tilde{X}$. If $q_{X}$ has $\operatorname{rank} N$ at $(a, \alpha)$, there is nothing to prove. Else $(a, \alpha) \in N_{X}$ and $d q\left(T_{(a, \alpha)} W_{X}\right)=T_{\alpha} H_{a} \supset T_{\alpha} X^{\vee}$. Since $L$ is transversal to $X^{\vee}$ at $\alpha$, it follows that $L$ is also transversal to $H_{a}$ at $\alpha$.

(3) The restriction of $f$ to $\tilde{Y}=Y \times L$ is just the second projection, so $f$ has no critical point in $\tilde{Y}$. Let $(a, \alpha) \in \tilde{X}$ be a critical point of $f$. The fact that $q: W_{X} \rightarrow \mathbb{P}^{*}$ is tranversal to $L$ implies that $(a, \alpha)$ is then also a singular point of $f$. So $(a, \alpha) \in N_{X}$. This implies that $\xi \in X^{\vee}$. As $\xi \in L$ also, $X^{\vee}$ must be smooth of dimension $N-1$ at $\xi$. But $N_{X} \cong N_{X \vee} \rightarrow X^{\vee}$ is an isomorphism over the smooth part of $X^{\vee}$ (why?), and so over $\alpha$ there is no other critical point than $(a, \alpha)$.

(4) Let $(a, \alpha) \in \tilde{X}$ be a critical point of $f$. We just saw that then $(a, \alpha) \in N_{X}$ and that $q \mid N_{X}$ has rank $N-1$ at $(a, \alpha)$. Choose projecive coordinates such that: $a=[1: 0: \cdots: 0], H_{\alpha}=\left\{x^{N}=0\right\}$ and $A=\left\{x^{0}=x^{N}=0\right.$. Now $T_{a} X \subset T_{a} H_{\alpha}$. Let $\left(t^{1}, \ldots, t^{n}\right)$ be local analytic coordinates for $X$ near $a$, so that $X \subset \mathbb{P}$ is given at $a$ by

$$
\left(t^{1}, \ldots, t^{n}\right) \rightarrow\left[1: x^{1}(t): \ldots x^{N}(t)\right]
$$

and $x^{N}$ has order $\geq 2$ at $t=0$. Parametrize $W_{X}$ near $(a, \alpha)$ by

$$
\left(t^{1}, \ldots, t^{n} ; u_{1}, \ldots, u_{N}\right) \rightarrow\left(\left[1: x^{1}(t): \cdots: x^{N}(t)\right],\left[g(t, u): u_{1}: \ldots ; u_{N-1}: 1\right]\right)
$$

where $g(t, u)=-\sum_{\nu=1}^{N-1} x^{\nu}(t) u_{\nu}-x^{N}(t)$. So in local coordinates, $f(t)=-x^{N}(t)=$ $g(t, 0)$. So we must show that

$$
\left(\frac{\partial^{2} g}{\partial t^{i} \partial t^{j}}(0,0)\right)
$$

is nonsingular.

To this end, we first observe that

$$
\left(\frac{\partial^{2} g}{\partial t^{i} \partial u_{\nu}}(0,0)\right)
$$

has rank $n$ since, by the explicit formula for $g$, it equals $\left(-\partial z^{\nu} / \partial t^{i}\right)$. This implies that the derivatives $\partial g / \partial t^{1}, \ldots, \partial g / \partial t^{n}$ are part of a system of local coordinates for $W$ at $(a, \alpha)$. But notice that their common zero set is precisely the locus where $q_{X}$ is not of maximal rank. By lemma (1.5) this is just $N_{X}$.

Since $q \mid N_{X}$ has rank $N-1$ it follows that the map

$$
\left(\partial g / \partial t^{1}, \ldots, \partial g / \partial t^{n}, g, u_{1}, \ldots, u_{N-1}\right)
$$


must have rank $n+N-1$ in $(0,0)$. As its jacobian at this point is

$$
\left(\begin{array}{cc}
\left(\partial^{2} g / \partial t^{i} \partial t^{j}\right)(0,0) & * \\
\left(\partial g / \partial t^{j}\right)(0,0)=-\left(\partial x^{N} / \partial t^{j}\right)(0)=0 & -x^{\nu}(0)=0 \\
0 & I_{N-1}
\end{array}\right)
$$

it follows that $\left(\partial^{2} g / \partial t^{i} \partial t^{j}\right)(0,0)$ is nonsingular.

The collection of hyperplane sections $X_{\xi}:=\left\{X \cap H_{\xi}\right\}_{\xi \in L}$ is called a general Lefschetz pencil; these sections are just the fibres of the map $f$, which itself is called a Lefschetz fibration. The subspace $A \subset \mathbb{P}$ is called its axis and $Y$ its fixed point locus. We will see that a general Lefschetz pencil is an algebro-geometric analogue of a Morse function on a manifold; it is an important tool to analyze the topology of $X$. 
10 E. Looijenga, Cohomology and intersection homology of algebraic varieties 


\section{LECTURE 2.}

\section{The weak Lefschetz theorem}

We will need some results from homotopy theory. We just state them here and refer for proofs to a standard text such as G.W. Whitehead's Elements of Homotopy theory (Springer Graduate Text in Mathematics). For the purposes of homotopy theory, the most suitable class of spaces to work with are those which are compactly generated, that is spaces that satisfy the Hausdoff axiom and have the property that a subset is closed iff its intersection with every compact subset is compact. We shall therefore assume that all spaces in this lecture belong to this class.

We say that a topological pair $(X, A)$ is a $k$-cellular extension if $X$ is obtained from $A$ by simultaneously attaching a number of $k$-cells. Such a pair is $(k-1)$ connected.

A relative cell complex of dimension $\leq d$ is a filtered space

$$
X_{\bullet}=\left\{X_{-1} \subset X_{0} \subset X_{1} \subset \cdots \subset X_{d}\right\}
$$

such that $\left(X_{k}, X_{k-1}\right)$ is a $k$-cellular extension for $k=0, \ldots, d ;\left(X_{d}, X_{-1}\right)$ is then said to admit the structure of a relative cell complex of dimension $\leq d$. If $X_{-1}=$ $\emptyset$, we omit the adjective relative. A large class of examples consist of the finite dimensional polyhedra with subcomplexes.

Relative cell complexes have several nice properties of which we list a few.

Proposition A. Let $(X, A)$ admit the structure of a relative cell complex and assume that $i: A \subset X$ is a homotopy equivalence. Then $A \subset X$ is a deformation retract, i.e., there exists a continuous family of maps $\left\{r_{t}: X \rightarrow X\right\}_{0 \leq t \leq 1}$ with $r_{t} i=i$ for all $t, r_{0}=\mathbf{1}_{X}$ and $r_{1}(X)=A$.

If $(X, A)$ admits the structure of a relative cell complex with $A=X_{k-1}$, then by iterated use of the homotopy exact sequence for triples we find that $(X, A)$ is $(k-1)$-connected. There is a homotopy converse which says that if $(X, A)$ admits the structure of a relative cell complex and is $(k-1)$-connected, then $(X, A)$ is homotopy equivalent relative $A$ to a pair $\left(X^{\prime}, A\right)$ which admits the structure of a relative cell complex with only cells in dimension $\geq k$. This practically implies the following two propositions. 
Proposition B. Let $f:(X, A) \rightarrow(Y, B)$ be a relative homeomorphism of pairs, i.e., $B$ is closed in $Y$ and $f$ maps $X-A$ homeomorphically onto $Y-B$. If $(X, A)$ is $(k-1)$-connected and admits the structure of a relative cell complex, then the same is true for $(Y, B)$.

Proposition C. Let $(X, A)$ resp. $(Y, B)$ be $(k-1)$-connected resp. $(l-1)$-connected and admit the structure of a relative cell complex. Then $(X, A) \times(Y, B):=(X \times$ $Y, X \times B \cup A \times Y)$ is $(k+l-1)$-connected.

This finishes our short homotopical review. The main result of this lecture is this:

(2.1) Theorem (Weak Lefschetz theorem). Let $X \subset \mathbb{P}$ be a closed smooth projective variety of dimension $n$, and let $X_{\xi}=X \cap H_{\xi}$ be a tranversal hyperplane section. Then

1. $\left(X, X_{\xi}\right)$ is $(n-1)$-connected and

2. $X-X_{\xi}$ has the homotopy type of a finite cell complex of dimension $\leq n$.

The second statement is actually a special case of the more general fact that any affine variety of complex dimension $\leq n$ has the homotopy type of a finite cell complex of dimension $\leq n$.

(2.2) Corollary. If $X$ is as in the previous theorem and $Z \subset \mathbb{P}$ is a linear subspace transversal to $X$, then $(X, X \cap Z)$ is $\operatorname{dim}(X \cap Z)$-connected.

Proof. Iterated application of part (1) of the weak Lefschetz theorem.

So $X$ is connected and of dimension $\geq 2$, then any tranversal linear section $X^{\prime} \subset X$ of dimension $\geq 1$ is also connected and induces a surjection on fundamental groups (even a bijection if $\operatorname{dim} X^{\prime} \geq 2$ ).

The proof of the weak Lefschetz theorem requires some preparation. We begin with recalling the Morse lemma:

(2.3) Lemma. If $f:\left(\mathbb{C}^{n}, 0\right) \rightarrow(\mathbb{C}, 0)$ has a non-degenerate singularity, then after a local-analytic coordinate change, $f$ takes the form $\sum_{i=1}^{n}\left(z^{i}\right)^{2}$.

(2.4) This motivates us to have a closer look at the function $f: \mathbb{C}^{n} \rightarrow \mathbb{C}$ defined by $f(z)=\sum_{i=1}^{n}\left(z^{i}\right)^{2}$.

Choose $\epsilon>0$ and let $B_{\epsilon}$ be the set $z \in \mathbb{C}^{n}$ with $|z| \leq \epsilon$. It is easily verified that $f \mid \partial B_{\epsilon}$ has no singular point $z$ with $|f(z)|<\epsilon^{2}$. Choose $0<\eta<\epsilon^{2}$, and let

$$
D:=\{t \in \mathbb{C}:|t| \leq \eta\}, \quad B:=f^{-1} D \cap B_{\epsilon}, \quad \dot{B}:=f^{-1} D \cap \partial B_{\epsilon} .
$$

From now on we reserve the symbols $f$ resp. $\dot{f}$ for the restrictions $f: B \rightarrow D$ resp. $f: \dot{B} \rightarrow D$. Notice that $\dot{f}$ is a proper smooth mapping without singularities and hence locally $C^{\infty}$ trivial. Since $D$ is a disk, $\dot{f}$ is then (globally) $C^{\infty}$ trivial.

We next turn our attention to the fibre $B_{\eta}:=f^{-1}(\eta)$. If $z=x+\sqrt{-1} y \in \mathbb{C}^{n}$, then $z \in B_{\eta}$ iff $|x|^{2}-|y|^{2}=\eta, x \cdot y=0$ and $|x|^{2}+|y|^{2} \leq \epsilon^{2}$. This implies that 
Figure 1 Thimble of an ordinary singularity

$|x|^{2}=|y|^{2}+\eta$ and $|y|^{2} \leq \frac{1}{2}\left(\epsilon^{2}+\eta\right)$. So if we make the substitutions

$$
u:=\frac{x}{|x|} \quad, \quad v:=\frac{y}{\sqrt{\frac{1}{2}\left(\epsilon^{2}+\eta\right)}},
$$

then $|u|=1,|v| \leq 1$ and $u \cdot v=0$. We thus obtain a diffeomorphism from $B_{\eta}$ onto the unit disk bundle of the tangent bundle of $S^{n-1}$.

The real part of $B$ is the set of $x \in \mathbb{R}^{n}$ with $|x|^{2} \leq \eta$; we denote it by $e$ and call it the thimble in $B$. Notice that $e \cap B_{\eta}=\partial e$ and that this intersection corresponds to zero section of $B_{\eta}$ under the above diffeomorphism.

(2.5) Lemma. The inclusions

$$
e \subset\left(B_{\eta} \cup e\right) \subset\left(\dot{B} \cup B_{\eta} \cup e\right) \subset B
$$

are all deformation retracts.

Proof. Since $e \cap B_{\eta} \subset B_{\eta}$ is a deformation retract, so is $e \subset B_{\eta} \cup e$. Since $\dot{f}$ is trivial, $\dot{B}_{\eta} \subset \dot{B}$ is a deformation retract. As $\dot{B} \cap\left(B_{\eta} \cup e\right)=\dot{B}_{\eta}$, it follows that the second inclusion is also a deformation retract. That the last inclusion has this property can probably be shown directly, but tedious work can be avoided by showing that $B$ is starlike and hence contractible. Since $e$ is contractible, so is $\dot{B} \cup B_{\eta} \cup e$, and hence the last inclusion is a homotopy equivalence. Now apply proposition (A).

We apply this lemma to the case when $f$ is part of a more global situation:

(2.6) Lemma. Suppose that we are given a compact $2 n$-manifold $Z$ with boundary $\partial Z$ which contains $B$ and a smooth mapping $F: Z \rightarrow D$ which extends $f$. We assume that $F^{-1}(\partial D)=\partial Z$, that $\dot{B}$ meets $\partial Z$ transversally, and that $F$ has no singular point $\neq 0$. Then $Z_{\eta} \cup e \subset Z$ is a deformation retract, so that up to homotopy, $Z$ is obtained from $Z_{\eta}$ by attaching an $n$-cell. 
Figure 2 Milnor's fibration

Proof. Put $\dot{Z}:=Z-(B-\dot{B})$. This is a manifold with corners. The restriction $\dot{F}: \dot{Z} \rightarrow D$ of $F$ has no critical points, even when we restrict to the boundary piece $\partial B$. The Ehresmann fibration theorem applies and we find that $\dot{F}$ is $C^{\infty}$ trivial. So $\dot{Z}_{\eta} \subset \dot{Z}$ is a deformation retract. Also, $\dot{Z}_{\eta} \subset \dot{Z}_{\eta} \cup \dot{B}$ is a deformation retract, because $\dot{B}_{\eta} \subset \dot{B}$ is. This implies that the inclusion $\dot{Z}_{\eta} \cup \dot{B} \subset \dot{Z}$ is a homotopy equivalence. Proposition (A) applies here, so this inclusion is actually a deformation retract. This in turn, implies that $B \cup Z_{\eta} \subset Z$ is a deformation retract. The lemma now follows if we combine this with the fact that $B_{\eta} \cup e \subset B$ is a deformation retract.

(2.7) Let us now put ourselves in the situation of the weak Lefschetz theorem. According to (1.7) there exists a line $L \subset \mathbb{P}$ passing through $\xi$ which is transversal to $X^{\vee}$. This defines a general Lefschetz pencil on $X$. We employ the notation of the previous lecture. So we have a Leschetz fibration $f: \tilde{X} \rightarrow X$ satisfying the properties listed in (1.8). If $\xi(1), \ldots, \xi(r)$ are the distinct points of $L \cap X^{\vee}$, then choose an affine coordinate $w$ on $L$ such that $w(\xi)=1$ and $|w(\xi(\rho))|<1$, $\rho=1, \ldots, r$. Let $L(0) \subset L$ and $L(\infty) \subset L$ be the hemispheres defined by $|w| \leq 1$ and $|w| \geq 1$ respectively. Let $(x(\rho), \xi(\rho))$ be the unique critical point of $f$ over $\xi(\rho)$. Choose (in terms of local analytic coordinates at this point for which is there a constant plus a sum of squares) a neighborhood $B(\rho)$ of $\xi(\rho)$ as in (2.4) whose image under $f$ is a closed disk $D(\rho)$ in the interior of $L(0)$ centered at $\xi(\rho)$. Let $\eta(\rho)$ the point of $D(\rho)$ with maximal real part so that the thimble $e(\rho)$ in $B(\rho)$ has its boundary $\partial e(\rho)$ in $B(\rho)_{\eta(\rho)}$. Draw arcs $\Gamma(\rho)$ in $L(0)$ connecting $\xi$ with $\eta(\rho), \rho=1, \ldots, r$, as in the picture below. Since $\tilde{X}$ is locally trivial over $\Gamma(\rho)$, the 
embedding $\partial e(\rho) \subset X_{\eta(\rho)}$ extends to an embedding of $\partial e(\rho) \times \Gamma(\rho)$ in $\tilde{X}_{\Gamma(\rho)}$ which commutes with the projection to $\Gamma(\rho)$. Let $\tilde{e}(\rho)$ denote the union of $e(\rho)$ and the image of this embedding. This is a $n$-cell whose boundary lies in $X_{\xi}$. Put

$$
X^{\#}:=X_{\xi} \cup \bigcup_{\rho=1}^{r} \tilde{e}(\rho) .
$$

This is an $n$-cellular extension and hence $(n-1)$-connected.

Figure 3 Deformation retract of $X_{L(0)}$

(2.8) Lemma. $X^{\#} \subset \tilde{X}_{L(0)}$ is a deformation retract.

Proof. Let $E$ be the union of the discs $D(\rho)$ and the $\operatorname{arcs} \Gamma(\rho)$. Then $E \subset L_{0}$ is a deformation retract. Since $f$ is locally trivial over $L(0)-\operatorname{int}(E)$, the homotopy lifting property assures that $\tilde{X}_{E} \subset X_{L(0)}$ is a deformation retract. According to (2.6), $X_{\eta(\rho)} \cup e(\rho) \subset \tilde{X}_{D(\rho)}$ is a deformation retract. A similar argument shows that

$$
X_{\xi} \cup \tilde{e}(\rho) \subset \tilde{X}_{D(\rho) \cup \Gamma(\rho)}
$$

is a deformation retract. The lemma then follows.

Proof of the weak Lefschetz theorem. We first show (1). For $n=0,1$ the statement is trivial. So we assume $n \geq 2$ and proceed by induction. We use the triple

$$
(A, B, C):=\left(\tilde{X}, \tilde{X}_{L(0)} \cup \tilde{Y}, X_{\xi} \cup \tilde{Y}\right) .
$$


Note that in

$$
(B, C)=\left(\tilde{X}_{L(0)} \cup \tilde{Y}, X_{\xi} \cup \tilde{Y}\right) \supset\left(\tilde{X}_{L(0)}, X_{\xi} \cup \tilde{Y}_{L(0)}\right) \supset\left(\tilde{X}_{L(0)}, X_{\xi}\right) \supset\left(X^{\#}, X_{\xi}\right)
$$

the first inclusion is a relative homeomorphism. The second is a relative homotopy equivalence since $\tilde{Y}$ is locally trivial over $L(0)$, and the third is a deformation retract by (2.8). Since $\left(X^{\#}, X_{\xi}\right)$ is $(n-1)$-connected, so is $(B, C)$.

Next observe that

$$
(A, C)=\left(\tilde{X}, X_{\xi} \cup \tilde{Y}\right) \rightarrow\left(X, X_{\xi}\right)
$$

is also a relative homeomorphism.

Finally, consider

$$
(A, B)=\left(\tilde{X}, \tilde{X}_{L(0)} \cup \tilde{Y}\right) \supset\left(\tilde{X}_{L(\infty)}, \tilde{X}_{\partial L(\infty)} \cup \tilde{Y}_{L(\infty)}\right) \cong\left(X_{\xi}, Y\right) \times(L(\infty), \partial L(\infty)),
$$

where the isomorphism comes from the local triviality of $f$ over $L(\infty)$. The induction hypothesis tells us that $\left(X_{\xi}, Y\right)$ is $(n-2)$-connected. Since $(L(\infty), \partial L(\infty))$ is 1-connected, it follows from proposition (C) that the product is $n$-connected. The inclusion in the above display is a relative homeomorphism. Proposition (B) applies, and so $(A, B)$ is also $n$-connected.

Feeding these properties in the exact homotopy sequence of the triple $(A, B, C)$ gives that $\left(X, X_{\xi}\right)$ is $(n-1)$-connected.

The proof of (2) also proceeds with induction, but this time starting with $n=0$. So assume that $n>0$. Note that all the maps in

$$
X-X_{\xi} \stackrel{\widetilde{F}}{\tilde{X}}-\tilde{Y}-X_{\xi} \cong \tilde{X}-\tilde{Y}-X_{w=\infty} \supset \tilde{X}_{L(0)}-\tilde{Y}_{L(0)} \subset X^{\#}-Y
$$

are homotopy equivalences. Now $\left(X^{\#}-Y, X_{\xi}-Y\right)$ is an $n$-cellular extension and by our induction hypothesis, $X_{\xi}-Y$ has the homotopy type of a finite cell complex of dimension $\leq n-1$. It follows that $X^{\#}-Y$, and hence $X-X_{\xi}$, has the homotopy type of a finite cell complex of dimension $\leq n$.

We now look at the homological aspects of the triple $(A, B, C)$ that appeared in the proof of the weak Lefschetz theorem. Our homotopy discussion and excision yield

$$
\begin{aligned}
& H_{k}(B, C)=H_{k}\left(X^{\#}\right)=\oplus_{\rho=1}^{r} H_{k}(\tilde{e}(\rho), \partial \tilde{e}(\rho))=\left\{\begin{array}{l}
0 \text { if } k \neq n ; \\
\cong \mathbb{Z}^{r} \text { else, }
\end{array}\right. \\
& H_{k}(A, C)=H_{k}\left(X, X_{\xi}\right) \\
& H_{k}(A, B)=H_{k}\left(\left(X_{\xi}, Y\right) \times(L(\infty), \partial L(\infty)) \cong H_{k-2}\left(X_{\xi}, Y\right),\right.
\end{aligned}
$$

where the last isomorphism follows from the Künneth formula. So the exact sequence of this triple becomes

$$
\rightarrow \oplus_{\rho} H_{k}(\tilde{e}(\rho), \partial \tilde{e}(\rho)) \rightarrow H_{k}\left(X, X_{\xi}\right) \stackrel{L^{\prime}}{\rightarrow} H_{k-2}\left(X_{\xi}, Y\right) \rightarrow \oplus_{\rho} H_{k-1}(\tilde{e}(\rho), \partial \tilde{e}(\rho)) \rightarrow
$$


The homomorphism $L^{\prime}$ can be geometrically understood as follows. Choose $\xi^{\prime} \in$ $L-X^{\vee}$ in a convex neighborhood $\xi$ (but $\neq \xi$ ). Then the intersection of $H_{\xi^{\prime}}$ with $\left(X, X_{\xi}\right)$ is transversal and gives the pair $\left(X_{\xi^{\prime}}, Y\right)$. Represent $z \in H_{k}\left(X, X_{\xi}\right)$ by a $k$-chain $Z$ which is transversal to $H_{\xi}$. Then its intersection with $H_{\xi^{\prime}}, Z \cdot H_{\xi^{\prime}}$, is a $(k-2)$-chain on $X_{\xi^{\prime}}$ with boundary in $Y$. Move $\xi^{\prime}$ to $\xi$ along a straight line and drag along $Z \cdot H_{\xi^{\prime}}$. This produces a cycle on $\left(X, X_{\xi^{\prime}}\right)$ representing $L^{\prime}(z)$.

\section{(2.9) Corollary.}

1. $H_{k}\left(X, X_{\xi}\right)=0$ if $k<n$,

2. $L^{\prime}: H_{k}\left(X, X_{\xi}\right) \rightarrow H_{k-2}\left(X_{\xi}, Y\right)$ is an isomorphism for $k \neq n, n+1$,

3. we have an exact sequence

$$
0 \rightarrow H_{n+1}\left(X, X_{\xi}\right) \stackrel{L^{\prime}}{\rightarrow} H_{n-1}\left(X_{\xi}, Y\right) \rightarrow \oplus_{\rho} H_{n}(\tilde{e}(\rho), \partial \tilde{e}(\rho)) \rightarrow H_{n}\left(X, X_{\xi}\right) \rightarrow 0
$$

Proof. (1) is immediate from the weak Lefschetz theorem, whereas (2) and (3) follow from the exact sequence above.

Another corollary is a formula for euler characteristics:

(2.10) Corollary. The euler characteristics of $X, X_{\xi}$ and $Y$ are related by

$$
e(X)=-e(Y)+2 e\left(X_{\xi}\right)+(-1)^{n} r
$$

where $r$ is the class of $X$.

Proof. Use the equalities

$$
\begin{aligned}
& e(B, C)=(-1)^{n} r, \\
& e(A, C)=e\left(X, X_{\xi}\right)=e(X)-e\left(X_{\xi}\right), \\
& e(A, B)=e\left(X_{\xi}, Y\right)=e\left(X_{\xi}\right)-\epsilon(Y),
\end{aligned}
$$

and the fact that the alternating sum of these numbers must be zero.

Example. Let $X$ be a smooth plane curve of degree $d$. Then $X_{\xi}$ is a finite set of $d$ points and $Y=\emptyset$. The class of $X$ is the degree of the dual curve, which is $d(d-1)$. We thus find $e(X)=-d^{2}+3 d$. Since $e(X)=2-2 g(X)$, this gives us the well-known formula for the genus: $g(X)=\frac{1}{2}(d-1)(d-2)$.

There are corresponding statements for cohomology:

(2.11) .

1. $H^{k}\left(X, X_{\xi}\right)=0$ if $k<n$,

2. $L^{\prime}: H^{k}\left(X_{\xi}, Y\right) \rightarrow H^{k+2}\left(X, X_{\xi}\right)$ is an isomorphism for $k \neq n, n+1$,

3. we have an exact sequence

$$
0 \rightarrow H^{n}\left(X, X_{\xi}\right) \rightarrow \oplus_{\rho} H^{n}\left(\tilde{e}(\rho), \partial \tilde{e}(\rho) \rightarrow H^{n-1}\left(X_{\xi}, Y\right) \stackrel{L^{\prime}}{\rightarrow} H^{n+1}\left(X, X_{\xi}\right) \rightarrow 0\right.
$$


in particular, $H^{n}\left(X, X_{\xi}\right)$ is torsion free.

We omit the proofs, since they are similar to their homological counterparts.

Application. Let $W \subset \mathbb{P}^{n}$ be a smooth hypersurface of degree $d$. Regard $W$ as a hyperplane section of $\mathbb{P}^{n}$ with respect to the $d$-fold Veronese embedding. Then

$$
\begin{aligned}
i \leq n-2: & H_{i}(W) \cong H_{i}\left(\mathbb{P}^{n}\right) \\
i=n-1: & H_{n-1}(W) \rightarrow H_{n-1}\left(\mathbb{P}^{n}\right) \text { is onto } \\
i \geq n: & H_{i}(W) \cong H^{2 n-2-i}(W) \cong H^{2 n-2-i}\left(\mathbb{P}^{n}\right) .
\end{aligned}
$$

It is well-known that $H_{i}\left(\mathbb{P}^{n}\right)$ and $H^{i}\left(\mathbb{P}^{n}\right)$ are equal to $\mathbb{Z}$ for $i=0,2,4, \ldots, 2 n$, and are zero otherwise. So $H_{n-1}(W)$ is really the only interesting homology group. The interesting part of that homology group is $V:=\operatorname{ker}\left(H_{n-1}(W) \rightarrow H_{n-1}\left(\mathbb{P}^{n}\right)\right.$. Clearly,

$$
e(W)=n-1+(-1)^{n-1} \operatorname{rk}(V),
$$

If we combine this formula with (2.10), then we see that $\operatorname{rk}(V)$, and hence the $(n-1)$ th Betti number of $X$ can be computed inductively. 


\section{LECTURE 3. The hard Lefschetz theorem}

(3.1) We shall need a few facts from algebraic topology. As some of them are not so standard, we list them here.

First, let $X$ be any space. The the cup product gives its total cohomology $H^{\bullet}(X)$ the structure of a graded-commutative ring with unit. The cap product gives its homology $H_{\bullet}(X)$ the structure of a graded unital module over $H^{\bullet}(X)$. So $1 \cap a=a$ and $\alpha \cap(\beta \cap a)=(\alpha \cup \beta) \cap a$. (We adopt the convention to denote homology classes by Latin letters and cohomology classes by Greek letters.) If $X$ is path connected, then $H_{0}(X)=\mathbb{Z}$, and the usual pairing of homology with cohomology can be written as

$$
\langle,\rangle: H^{k}(X) \times H_{k}(X) \stackrel{\cap}{\longrightarrow} H_{0}(X)=\mathbb{Z} .
$$

The universal coefficient theorem for cohomology implies that this pairing is perfect if we mod out the torsion.

The preceding notions are functorial: if $f: X \rightarrow Y$ is a continuous map of spaces, then

$$
f^{*}: H^{\bullet}(Y) \rightarrow H^{\bullet}(X)
$$

is a homomorphism of graded-commutative unital rings, and

$$
f_{*}: H^{\bullet}(X) \rightarrow H^{\bullet}(Y)
$$

is homomorphism of $H^{*}(Y)$-modules. So

$$
f_{*}\left(f^{*} \omega \cap a\right)=\omega \cap f_{*} a .
$$

Now let $M$ be a compact $m$-manifold which is oriented by means of an orientation (=fundamental) class $[M] \in H_{m}(M)$. Then

$$
\cap[M]: H^{k}(M) \rightarrow H_{m-k}(M)
$$


is an isomorphism (Poincaré duality). This gives rise to the intersection pairing

$$
H_{m-k}(M) \times H_{k}(M) \cong H^{k}(M) \times H_{k}(M) \stackrel{<,}{\longrightarrow} \mathbb{Z}, \quad(a, b) \mapsto a \cdot b .
$$

Suppose now that $P$ is another compact oriented manifold, of dimension $p$ and let $f: M \rightarrow P$ be continuous. Put $c:=p-m$. Define the Gysin homomorphism as the composite

$$
f_{!}: H^{k}(M) \stackrel{P D}{\cong} H_{m-k}(M) \stackrel{f_{*}}{\longrightarrow} H_{m-k}(P) \stackrel{P D}{\cong} H^{k+c}(P)
$$

This map is adjoint to $f^{*}$ in the sense that

$$
f_{!} \alpha \cdot \zeta=\alpha \cdot f^{*} \zeta
$$

(We could have defined $f_{1}$ this way if we were working with rational coefficients.) The map $f_{!}$is also a $H^{\bullet}(P)$-homomorphism:

$$
f_{!}\left(f^{*} \zeta \cup \alpha\right)=\zeta \cup f_{!} \alpha
$$

We will only need the special case $\alpha=1$ :

$$
f_{1} f^{*} \zeta=\zeta \cup f_{1}(1)
$$

The class $f_{1}(1) \in H^{c}(P)$ is easy to understand geometrically in case $f$ is an embedding: it is then represented by the cocycle which assigns to an $M$-tranversal $c$-chain its intersection product with $M$. The Gysin maps behave well under transversal restriction, and are functorial with respect to compositions. This implies for instance, that if $i_{1}: M_{1} \subset P$ and $i_{2}: M_{2} \subset P$ are mutually transversal submanifolds with intersection $i: M \subset P$, then

$$
i_{!}(1)=i_{1 !}(1) \cup i_{2 !}(1) .
$$

The corresponding dual notion is

$$
f^{!}: H_{k}(P) \stackrel{P D}{\cong} H^{p-k}(P) \stackrel{f^{*}}{\longrightarrow} H^{p-k}(M) \stackrel{P D}{\cong} H_{k-c}(M)
$$

which is adjoint to $f_{*}$. We have

$$
f_{*}\left(\alpha \cap f^{!} z\right)=(-1)^{c} f_{!}(\alpha) \cap z,
$$

so that in particular

$$
f_{*} f^{!} z=(-1)^{c} f_{!}(1) \cap z .
$$

This finishes our review of this bit of algebraic topology. 
We first illustrate these notions by means of a simple example. If $i: H \subset \mathbb{P}$ is a hyperplane, then we have defined $i_{H !}(1) \in H^{2}(\mathbb{P})$. Since all such hyperplane sections are isotopic, this class does not depend of the choice $H$; it is called the hyperplane class, and we shall denote it by $\eta$.

(3.2) Lemma. Let $i_{Z}: Z \subset \mathbb{P}$ be a linear subspace of codimension $k$. Then $i_{Z !}(1)=\eta^{k}$.

Proof. For $k=1$ there is nothing to prove. We proceed with induction on $k$, and assume $k \geq 2$. Then $Z$ is of the form $Z^{\prime} \cap H$, where $Z^{\prime}$ is of codimension $k-1$, it follows that

$$
i_{Z !}(1)=i_{Z^{\prime}}(1) \cup i_{H}(1)=\eta^{k-1} \cup \eta=\eta^{k} .
$$

Remarks. 1. It is well-known that $H^{\bullet}(\mathbb{P})$ is additively generatered by $1, \eta, \ldots \eta^{n}$.

2. If $X \subset \mathbb{P}$ is a closed smooth subvariety and $Z \subset X$ is an $X$-transversal codimension $k$ linear subspace, and $i: X \cap Z \subset X$ the corresponding linear section, then the preceding lemma implies that $i_{1}(1)=\eta_{X}^{k}$, where $\eta_{X}$ is the restriction of $\eta$ to $X$.

(3.3) We return to the situation studied in the previous lecture, where we are given $\mathbb{P} \supset X \supset X_{\xi} \supset Y$. Recall that for every extended thimble $\tilde{e}(\rho)$, its boundary is an embedded sphere in $X_{\xi}$. If we pick an orientation of the thimble, then its boundary gets also orientated, and may thus be regarded as an $(n-1)$-cycle on $X_{\xi}$. This is called a vanishing cycle. The submodule of $H_{n-1}\left(X_{\xi}\right)$ generated by these cycles is called the vanishing homology (of the pair $\left(X, X_{\xi}\right)$ ); we shall denote it by $V$. Put

$$
(u, \partial u): \cup_{\rho}(\tilde{e}(\rho), \partial \tilde{e}(\rho)) \rightarrow\left(X, X_{\xi}\right),
$$

and look at the commutative diagram below:

$$
\begin{array}{cccc}
\oplus_{\rho} H_{n}(\tilde{e}(\rho), \partial \epsilon(\rho)) & \stackrel{\partial_{*}}{\longrightarrow} & \oplus_{\rho} \tilde{H}_{n-1}(\partial \tilde{e}(\rho) \\
\downarrow u_{*} & & & \partial u_{*} \downarrow \\
H_{n}\left(X, X_{\xi}\right) & \stackrel{\partial_{*}}{\longrightarrow} & H_{n-1}(X-\xi) .
\end{array}
$$

The vanishing homology is just the image of the right vertical arrow $\partial u_{*}$. It follows from (2.10) that the left vertical arrow $u_{*}$ is surjective. Since the upper boundary map is an isomorphism, it follows that $V$ is equal to the image of the lower boundary map. In particular, $V$ only depends on the pair $\left(X, X_{\xi}\right)$.

Now consider the diagram

$$
\begin{aligned}
& H_{n}\left(X, X_{\xi}\right) \stackrel{\partial_{\varkappa}}{\longrightarrow} \underset{\|}{H_{n-1}\left(X_{\xi}\right) \stackrel{i_{\varkappa}}{\longrightarrow}} H_{n-1}(X) \quad \longrightarrow 0 \\
& H_{n-1}\left(X_{\xi}\right) \stackrel{i^{!}}{\longleftarrow} \quad H_{n+1}(X) \\
& P D \uparrow \cong \quad P D \uparrow \cong \\
& H^{n}\left(X, X_{\xi}\right) \stackrel{\partial^{*}}{\longleftarrow} H^{n-1}\left(X_{\xi}\right) \stackrel{i^{*}}{\longleftarrow} H^{n-1}(X) \longleftarrow 0 .
\end{aligned}
$$


The upper and lower rows are exact (the zeroes in these sequences come from the vanishing of $H_{n-1}\left(X, X_{\xi}\right)$ and $\left.H^{n-1}\left(X, X_{\xi}\right)\right)$. We see that $V=\operatorname{ker}\left(i_{*}\right)$. We define the invariant homology (of the pair $\left(X, X_{\xi}\right)$ ) as the image of $i^{!}: H_{n+1}(X) \rightarrow$ $H_{n-1}\left(X_{\xi}\right)$. These are the classes which are representable as intersections of $X_{\xi}$ with $X_{\xi}$-transversal $(n+1)$-cycles on $X$.

(3.4) Lemma. A class in $H_{n-1}\left(X_{\xi}\right)$ is invariant iff its intersection product with every vanishing cycle is zero.

Proof. Let $V^{\perp}$ denote the set of classes in $H_{n-1}\left(X_{\xi}\right)$ whose intersection product with every vanishing cycle is zero. Since $i^{!}$is the adjoint of $i_{*}$ the image of $i^{!}$and the perp of ker $i_{*}$ coincide after tensoring with $\mathbb{Q}$. This means that $I \subset V^{\perp}$ and that the quotient is torsion. But this quotient can be identified with a subgroup of $H^{n}\left(X, X_{\xi}\right)$, which, by (2.11), is torsion free. So $I=V^{\perp}$.

(3.5) Proposition. The following six properties are equivalent:

1. $V_{\mathbb{Q}} \cap I_{\mathbb{Q}}=0$,

2. $H_{n-1}\left(X_{\xi} ; \mathbb{Q}\right)=V_{\mathbb{Q}} \oplus I_{\mathbb{Q}}$.

3. $V_{\mathbb{Q}}$ is a nondegenerate subspace of $H_{n-1}\left(X_{\xi} ; \mathbb{Q}\right)$,

4. $I_{\mathbb{Q}}$ is a nondegenerate subspace of $H_{n-1}\left(X_{\xi} ; \mathbb{Q}\right)$,

5. $i_{*}$ maps $I_{\mathbb{Q}}$ isomorphically onto $H_{n-1}(X ; \mathbb{Q})$,

6. $i_{*} i^{!}: H_{n+1}(X ; \mathbb{Q}) \rightarrow H_{n-1}(X ; \mathbb{Q})$ is an isomorphism.

Proof. The mutual equivalence of the first four properties is elementary.

To see that $(1) \Leftrightarrow(5)$, observe that $\operatorname{dim} H_{n-1}(X ; \mathbb{Q})=\operatorname{dim} I_{\mathbb{Q}}$ (see the large diagram in (3.3)). Then note that $V_{\mathbb{Q}} \cap I_{\mathbb{Q}}$ is the kernel of $i_{*} \mid I_{\mathbb{Q}}$

Finally, $i_{*} \mid I_{\mathbb{Q}}$ is an isomorphism iff $i_{*} i^{!}$is.

(3.6) theorem (Hard Lefschetz theorem). The equivalent assertions of the preceding proposition are all true.

Lefschetz asserted this theorem in his famous monograph l'Analysis Situs et la Géometrie Algebrique (1924). It is called this way, because no one has been able to understand his (geometric) proof. The first accepted proof was not geometric, but analytic in character (based on the harmonic representation of the complex cohomology), and is largely due to Hodge. Another proof was given in the seventies by Deligne [7]. He first proves it for varieties defined over a finite field, and then invokes a comparison theorem to pass to the characteristic zero case. It is geometric, but in a rather roundabout way. In this course we shall follow a third approach (mainly due to P. Deligne, S. Zucker and M. Saito) which uses a mixture of geometric and analytic tools. It has the advantage that it is prototypical for a generalization to the case of singular varieties (with intersection homology replacing homology). In the remainder of this lecture we derive some consequences of the hard Lefschetz theorem (we indicate this dependence by an asterisk).

(3.7) Theorem* For $k \geq 0$, the maps

$$
\eta_{X}^{k} \cap: H_{n+k}(X ; \mathbb{Q}) \rightarrow H_{n-k}(X ; \mathbb{Q}), \quad \eta_{X}^{k} \cup: H^{n-k}(X ; \mathbb{Q}) \rightarrow H^{n+k}(X ; \mathbb{Q})
$$


are isomorphisms.

In more geometric terms: intersecting $(n+k)$-cycles on $X$ transversally with a fixed codimension $k$ linear subspace of $\mathbb{P}$ defines an isomorphism of $H_{n+k}(X ; \mathbb{Q})$ onto $H_{n-k}(X ; \mathbb{Q})$.

Proof. The second assertion follows from the first by applying Poincaré duality, so we only need to prove the first assertion. For this we choose transversal linear sections $X_{0} \subset X_{1} \subset X$ of dimension $n-k$ resp. $n-k+1$. Name the inclusions as follows: $i: X_{0} \subset X_{1}, i_{1}: X_{1} \subset X, i_{0}=i_{1} i: X_{0} \subset X$. Then $\eta_{X}^{k}=i_{0 !}(1)$, and so $\eta_{X}^{k} \cap=i_{0 !}(1) \cap=i_{0 *} i_{0}^{!}=i_{1 *} i_{*} i^{!} i_{1}^{!}$. If we compose $i^{1 !}: H_{n+k}(X) \rightarrow H_{n-k+2}\left(X_{1}\right)$ on both sides with the Poincaré duality isomorphisms we get by definition the natural map $i_{1}^{*}: H^{n-k}(X) \rightarrow H^{n-k}\left(X_{1}\right)$, and (2.11) implies that this is an isomorphism. Similarly, (2.9) implies that $i_{1 *}: H_{n-k}\left(X_{1}\right) \rightarrow H_{n-k}(X)$ is an isomorphism. It remains to see that $i_{*} t^{1}$ is an isomorphism, at least if we tensor with $\mathbb{Q}$. But this is just equivalent to the hard Lefschetz theorem for $X_{1}$ and its hyperplane section $\left.X_{0}\right)$.

Let us simply write $H^{r}$ for $H^{r}(X, \mathbb{Q})$ and $\eta$ for $\eta_{X}$. Define for $k \geq 0$ a bilinear $(-)^{n-k}$-symmetric form on $H^{n-k}$ by

$$
\Psi: H^{n-k} \times H^{n-k} \rightarrow \mathbb{Q}, \quad \Psi(\alpha, \beta):=\left\langle\alpha \cup \beta \cup \eta^{k},[X]\right\rangle .
$$

The preceding theorem implies that $\Psi$ is nondegenerate. Note also that $\eta \cup$ : $H^{n-k-2} \rightarrow H^{n-k}$ respects this form, so that its image will be a nondegenerate subspace.

We now define the primitive cohomology (resp. primitive homology) by

$$
\begin{aligned}
& P^{n-k}(X):=\operatorname{ker}\left(\eta^{k+1} \cup: H^{n-k} \rightarrow H^{n+k+2}\right), \\
& P_{n+k}(X):=\operatorname{ker}\left(\eta^{k+1} \cap: H_{n+k} \rightarrow H_{n-k-2}\right) .
\end{aligned}
$$

(3.8) Lemma*. $P^{n-k}(X)$ is the perp of $\eta \cup H^{n-k-2}$ with respect to $\Psi$; in particular, it is a nondegenerate subspace of $H^{n-k}$.

Proof. Let $\alpha \in H^{n-k}$. Then $\alpha \in P^{n-k}(X) \Leftrightarrow \alpha \cup \eta^{k+1}=0 \Leftrightarrow \alpha \cup \eta^{k+1} \cup \beta=0$ for all $\beta \in H^{n-k-2} \Leftrightarrow \Psi(\alpha, \eta \cup \beta)=0$ for all $\beta \in H^{n-k-2}$.

(3.9) Corollary* (Lefschetz decomposition). We have a natural isomorphism of $\mathbb{Q}[\eta]$-modules

$$
H^{\bullet}(X ; \mathbb{Q}) \cong \oplus_{k=0}^{n} P^{n-k}(X)[\eta] /\left(\eta^{k+1}\right) .
$$

This decomposition is orthogonal with respect to $\Psi$.

Proof. In view of (3.7) it suffices to show that for $0 \leq q \leq n$ we have an orthogonal decomposition

$$
\left.H^{q} X ; \mathbb{Q}\right)=P^{q}(X) \perp\left(\eta \cup P^{q-2}(X)\right) \perp\left(\eta^{2} \cup P^{q-4}(X)\right) \perp \cdots
$$

But this follows easily from the preceding lemma. 
Remark. The endomorphism of $H^{\bullet}(X ; \mathbb{Q})$ defined by $\eta_{X} \cup$ is often denoted by $L$. If $B$ is the endomorphism of $H^{\bullet}(X ; \mathbb{Q})$ which multiplies classes in degree $q$ by $n-q$, then it is easily checked that $[L, B]=-2 L$. The Lefschetz decomposition implies the existence and uniqueness of an operator $\Lambda$ of degree -2 with the property that $[\Lambda, L]=B$. This means that the $\mathbb{Q}$-span of $L, B$ and $\Lambda$ is closed under the bracket, and that as a Lie algebra it is isomorphic to $s l_{2}(\mathbb{Q})$ via

$$
\Lambda \leftrightarrow\left(\begin{array}{ll}
0 & 1 \\
0 & 0
\end{array}\right) \quad B \leftrightarrow\left(\begin{array}{cc}
1 & 0 \\
0 & -1
\end{array}\right) \quad L \leftrightarrow\left(\begin{array}{cc}
0 & 0 \\
-1 & 0
\end{array}\right) .
$$

Thus $H^{\bullet}(X ; \mathbb{Q})$ becomes a representation space of $s l_{2}(\mathbb{Q})$. The summands of the Lefschetz decomposition are just its isotypical components: the subrepresentation generated by any nonzero $a \in P^{n-k}(X)$ is irreducible of dimension $k+1$ (and equal to the span of $\left.a, \eta \cup a, \ldots \eta_{X}^{k} \cup_{X} a\right)$. It is not known whether there exists an algebro-geometric definition for $\Lambda$ as there is for $L$ (more precisely, the question is whether $\Lambda$ can defined by an algebraic cycle on $X \times X)$. One of Grothendieck's 'standard' conjectures asserts that this should be the case. 


\section{LECTURE 4. Monodromy}

(4.1) Suppose we are given a locally trivial map of topological spaces $f: E \rightarrow$ $B$. If $\gamma:[0,1] \rightarrow B$ is a path from $p \in B$ to $q \in B$, then the pull-back $\gamma^{*} E \rightarrow[0,1]$ is also locally trivial. A finite number of local trivializations of this map cover $[0,1]$ and they can be composed to give a global trivialisation. This defines a continuous family of homeomorphisms $\left\{h_{t}: E_{p} \rightarrow E_{\gamma(t)}\right\}_{0 \leq t \leq 1}$ such that $h_{0}$ is the identity. The family is not unique, but the isotopy class of each $h_{t}$ is. In particular, we get a well-defined isotopy class of homeomorphisms $E_{p} \rightarrow E_{q}$. Changing $\gamma$ within its homotopy class (modulo its end points), does not change this isotopy class. Furthermore, composition of homotopy classes of paths corresponds to composition of isotopy classes. In particular, we get a homomorphism from the fundamental group $\pi_{1}(B, p)$ to the group of isotopy classes of self-homeomorphisms of $E_{p}$ (we adopt the convention that the product $u v$ in $\pi_{1}(B, p)$ stands for: traverse first $v$, then $u$.) This homomorphism is called the geometric monodromy of $f$ relative $p$. Isotopy classes of of self-homeomorphisms of $E_{p}$ act on the homology and the cohomology of $E_{p}$. The ensueing representation of $\pi_{1}(B, p)$ on $H_{k}\left(E_{p}\right)$ or $H^{k}\left(E_{p}\right)$ is called the monodromy representation (of $f$ relative $p$ ).

Let us now consider the special case that $B$ is the unit circle $S^{1}$. So if $F$ denotes the fiber $E_{1}$, then we can find a continuous family of homeomorphisms $\left\{h_{\theta}: F \rightarrow E_{\exp (\sqrt{-1} \theta)}\right\}_{0 \leq \theta \leq 2 \pi}$, such that $h_{0}$ is the identity and $h:=h_{2 \pi}$ represents the geometric monodromy corresponding to the natural generator of $\pi_{1}\left(S^{1}, p\right)$; in this case we will simply refer to $h$ as the geometric monodromy of $f$.

Suppose now that we are given a subspace $\dot{E} \subset E$ plus a trivialisation $u$ : $\dot{E} \rightarrow \dot{F}$ of $f \mid \dot{E}$. Assume that the local trivialisations of $f$ can be chosen to be compatible with $u$. Such local trivialisations will yield an $h$ which is the identity on $\dot{F}$. Its relative isotopy class relative $\dot{F}$ is again independent of choices. This relative isotopy class remains unaltered if we move $u$ inside the same homotopy class of trivialisations. There is now a well-defined action of $h$ on the long exact (co)homology sequence of the pair $(F, \dot{F})$. But we can extract finer homological information out of $h$ : if $z$ is a $k$-chain on $F$ whose boundary is supported by $\dot{F}$, then $h_{*}(z)$ has the same boundary as $z$, so that $h_{*}(z)-z$ is a $k$-cycle. This induces a homomorphism

$$
\operatorname{var}(h)_{*}: H_{k}(F, \dot{F}) \rightarrow H_{k}(F)
$$


called the variation homomorphism. It determines the action of $h$ on both $H_{k}(F)$ and $H_{k}(F, \dot{F})$, for if $j_{*}: H_{k}(F) \rightarrow H_{k}(F, \dot{F})$ is the natural map, then these actions are given by $\operatorname{var}(h)_{*} j_{*}$ and $j_{*} \operatorname{var}(h)_{*}$ respectively.

(4.2) Now let us go back to the model $f: B \rightarrow D$ of the quadratic function introduced in (2.4). In the discussion that follows we are more concerned with the fibers of $f$ then with its total space, which is why we find it convenient to let $n$ denote the fiber dimension (so that $B$ has complex dimension $n+1$ ). We will also assume that $n \geq 1$. Recall that $f \mid \dot{B}$ is trivial. A trivialisation of $f \mid \dot{B}$ is unique up to homotopy (because $D$ is contractible), and so we have a well-defined isotopy class of trivialisations of $\dot{B}_{\partial D} \rightarrow \partial D$. The $\operatorname{map}\left(B_{\partial D}, \dot{B}_{\partial D}\right) \rightarrow \partial D$ is a locally trivial fibration of manifolds with boundary, and local trivialisations can be chosen to be compatible with a given trivialisation of $\dot{B}_{\partial D} \rightarrow \partial D$. So the preceding discussion applies to this situation and we find a geometric monodromy $h: B_{\eta} \rightarrow B_{\eta}$ which is the identity on $\dot{B}_{\eta}$. In (2.4) we constructed a diffeomorphism of $B_{\eta}$ onto the unit disk bundle of the $n$-sphere. Via this diffeomorphism one can give an explicit representative of $h$ (cf. Lamotke [12]), but we shall not do this here. We content ourselves with a formula for the associated variation homomorphism. If we orient the thimble $e$, we get an orientation of its boundary; we denote the corresponding (vanishing) cycle by $\Delta$ and its class in $H_{n}\left(B_{\eta}\right)$ by $[\Delta]$. With respect to the natural orientation of the tangent bundle of $S^{n}$, the self-intersection of its zero-section is just the Euler characteristic of $S^{n}$, i.e., $1+(-1)^{n}$. A short computation shows that the diffeomorphism of $B_{\eta}$ onto the unit disk bundle in $T S^{n}$ has degree $(-1)^{\frac{1}{2} n(n-1)}$, so that

$$
[\Delta] \cdot[\Delta]=(-1)^{\frac{1}{2} n(n-1)}\left(1+(-1)^{n}\right) .
$$

It is clear that $H_{k}\left(B_{\eta}\right)$ is trivial for $k \neq 0, n$ and is freely generated by [ $\left.\Delta\right]$ for $k=n$. Let $\Delta^{*}$ be an $n$-chain on $B_{\eta}$ which under the diffeomorphism corresponds to a fibre of the unit disk bundle over $S^{n}$, and is oriented in such a way that $\Delta \cdot \Delta^{*}=1$ (the dot product is here taken with respect to the complex orientation of $\left.B_{\eta}\right)$. We let $\left[\Delta^{*}\right]$ denote its class in $H_{n}\left(B_{\eta}, \dot{B}_{\eta}\right)$. We have $H_{k}\left(B_{\eta}, \dot{B}_{\eta}\right) \cong H^{2 n-k}\left(\left(B_{\eta}\right)\right.$ and so this group is zero for $k \neq n, 2 n$, and is freely generated by [ $\left.\Delta^{*}\right]$ for $k=n$. So the variation homomorphism $\operatorname{var}(h)_{*}: H_{k}\left(B_{\eta}, \dot{B}_{\eta}\right) \rightarrow H_{k}\left(B_{\eta}\right)$ is necessarily trivial for $k \neq n$. For $k=n$ it must map $[\Delta]^{*}$ to a scalar multiple of $[\Delta]$. The coefficient was computed by Lefschetz:

(4.3) Proposition. $\operatorname{var}(h)_{*}\left[\Delta^{*}\right]=-(-1)^{\frac{1}{2} n(n-1)}[\Delta]$.

Now let us put ourselves in a situation comparible to that of lemma (2.6): we assume we are given a space $Z$ containing $B$ and a continuous mapping $F: Z \rightarrow D$ extending $f$. It is assumed that the pair $(\dot{Z}:=Z-(B-\dot{B}), \dot{B})$ is locally trivial over $D$. We further assume that the situation is reasonable: we want the inclusion $\left(B_{\eta}, \dot{B}_{\eta}\right) \subset\left(Z_{\eta}, \dot{Z}_{\eta}\right)$ to be excisive. Since $F$ is locally trivial over $\partial D$, there is defined a geometric monodromy $\sigma: Z_{\eta} \rightarrow Z_{\eta}$. We can (and will) take it to be $h$ on $B_{\eta}$ and the identity on $\dot{Z}_{\eta}$. 
(4.4) Corollary (The Picard-Lefschetz formula's). The monodromy $\sigma$ acts on the cohomology of $Z_{\eta}$ as the identity in degrees $\neq n$; in degree $n$ it is given by

$$
\sigma_{*}(a)=a-(-1)^{\frac{1}{2} n(n-1)}(\delta \cdot a) \delta \quad \text { resp. } \quad \sigma^{*}(\alpha)=\alpha-(-1)^{\frac{1}{2} n(n-1)}\langle\delta, \alpha\rangle \delta^{*},
$$

where $\delta \in H_{n}\left(Z_{\eta}\right)$ denotes the class of $\Delta$ in $H_{n}\left(Z_{\eta}\right)$, and $\delta^{*} \in H^{n}\left(Z_{\eta}\right)$ is the class that assigns to a $\Delta$-transversal $n$-cycle $z$ the value $\Delta \cdot z$.

Proof. We can factorize $\sigma_{*}-1$ as follows:

$$
H_{k}\left(Z_{\eta}\right) \rightarrow H_{k}\left(Z_{\eta}, \dot{Z}_{\eta}\right) \cong H_{k}\left(B_{\eta}, \dot{B}_{\eta}\right) \stackrel{\operatorname{var}(h)_{*}}{\longrightarrow} H_{k}\left(B_{\eta}\right) \rightarrow H_{k}\left(Z_{\eta}\right)
$$

where the extremal maps are the natural ones. Since $\operatorname{var}(h)_{*}$ is trivial in degree $k \neq n$, it follows that $\sigma_{*}$ is the identity in these degrees. Now let $a \in H_{n}\left(Z_{\eta}\right)$. Its image in $H_{k}\left(B_{\eta}, \dot{B}_{\eta}\right)$ will be multiple $\lambda$ of $\left[\Delta^{*}\right]$. Using $[\Delta] \cdot\left[\Delta^{*}\right]=1$, we see that $\lambda=[\Delta] \cdot \lambda\left[\Delta^{*}\right]=\delta \cdot a$. According to (4.2) the image of $\left[\Delta^{*}\right]$ in $H_{n}\left(Z_{\eta}\right)$ is equal to $-(-1)^{\frac{1}{2} n(n-1)} \delta$. The formula for $\sigma_{*}$ now follows. The proof of the formula for $\sigma^{*}$ is similar.

(4.5) In the case that $Z$ is part of a complex $(n+1)$-manifold, then the dot product on $\left.H_{(} Z_{\eta}\right)$ is preserved by the monodromy (for $\sigma$ respects the complex orientation). This dot product is symmetric for even $n$ and skew for odd $n$. When $n$ is even, $\delta, \delta= \pm 2$ (in particular, $\delta$ is not a torsion class) and $\sigma_{*}$ is the reflection with respect to $\delta$ relative the dot product. When $n$ is odd, the dot product is skew and $\sigma_{*}$ is a symplectic transvection; in this case it could happen that $\delta$ is torsion.

(4.6) It is not difficult to deduce from our assumptions that $Z_{0} \subset Z$ is a deformation retract. We also know that $Z_{\eta} \cup e \subset Z$ is a deformation retract, so that $H_{k}\left(Z, Z_{\eta}\right) \cong H_{k}(e, \partial e) \cong \tilde{H}_{k-1}(\partial e)$ is trivial for $k \neq n+1$ and free of rank one for for $k=n$ (in that case, $[\Delta]$ determines a generator). If we substitute this in the exact sequence of the pair $\left(Z, Z_{\eta}\right)$ we get:

$$
H_{k}\left(Z_{0}\right) \cong H_{k}(Z) \cong H_{k}\left(Z_{\eta}\right), \text { for } k \neq n, n+1 \text {. }
$$

and an exact sequence

$$
0 \rightarrow H_{n+1}\left(Z_{\eta}\right) \rightarrow H_{n+1}\left(Z_{0}\right) \rightarrow \mathbb{Z} \rightarrow H_{n}\left(Z_{\eta}\right) \rightarrow H_{n}\left(Z_{0}\right) \rightarrow 0
$$

The image of the generator of $\mathbb{Z}$ in $H_{n}\left(Z_{\eta}\right)$ is $\delta$. It follows that $H_{n}\left(Z_{0} ; \mathbb{Q}\right)$ can be identified with the cokernel of $\sigma_{*}: H_{n}\left(Z_{\eta} ; \mathbb{Q}\right) \rightarrow H_{n}\left(Z_{\eta} ; \mathbb{Q}\right)$. This is the largest quotient of $H_{n}\left(Z_{\eta} ; \mathbb{Q}\right)$ on which $\sigma_{*}$ acts trivially, and is for that reason called the space of co-invariants of $\sigma$ in $H_{n}\left(Z_{\eta} ; \mathbb{Q}\right)$.

We now distinguish two cases according to whether $\delta$ is torsion:

The usual case: $\delta_{\mathbb{Q}} \neq 0$. Then we have a short exact sequence

$$
0 \rightarrow \mathbb{Q} \delta \rightarrow H_{n}\left(Z_{\eta} ; \mathbb{Q}\right) \rightarrow H_{n}\left(Z_{0} ; \mathbb{Q}\right) \rightarrow 0
$$


and $H_{n+1}\left(Z_{0} ; \mathbb{Q}\right) \cong H_{n+1}\left(Z_{\eta} ; \mathbb{Q}\right)$. So $H_{\bullet}\left(Z_{0} ; \mathbb{Q}\right)$ can be identified with the space of $\sigma$-co-invariants in $H_{\bullet}\left(Z_{\eta} ; \mathbb{Q}\right)$

The special case: $\delta_{\mathbb{Q}}=0$. This can only happen when $n$ is odd. Then $H_{n}\left(Z_{\eta} ; \mathbb{Q}\right)$ $\cong H_{n}\left(Z_{0} ; \mathbb{Q}\right)$ and we have a short exact sequence

$$
0 \rightarrow H_{n+1}\left(Z_{\eta} ; \mathbb{Q}\right) \rightarrow H_{n+1}\left(Z_{0} ; \mathbb{Q}\right) \rightarrow H_{n+1}(e, \partial \ell ; \mathbb{Q}) \rightarrow 0
$$

There is parallel cohomological discussion which we do not bother to explicate. For instance,

$$
H^{k}\left(Z_{0}\right) \cong H^{k}(Z) \cong H_{k}\left(Z_{\eta}\right), \text { for } k \neq n, n+1,
$$

and $H^{n}\left(Z_{0} ; \mathbb{Q}\right)$ can be identified with the space of $\sigma$-invariants in $H^{n}\left(Z_{\eta} ; \mathbb{Q}\right)$.

(4.7) We can now explain why in (3.3) the image of $i^{!}$was called the invariant homology. Let in the situation of $(2.7) \tilde{\delta}(\rho) \in H_{n-1}\left(X_{\xi}\right)$ be the class defined by an orientation of $\partial \tilde{e}(\rho)$. Put $U:=L-X^{\vee}$, and let $\gamma(\rho)$ be the loop in $U$ based at $\xi$ that traverses first $\Gamma(\rho)$ from $\xi$ to $\eta(\rho)$, next encircles $\xi(\rho)$ along $\partial D(\rho)$ in the counter clockwise direction and then goes back again to $\xi$ via $\Gamma(\rho)$. The monodromy along $\gamma(\rho)$ can only be nontrivial in degree $n$, and in that degree it is given by $a \mapsto a \pm(\tilde{\delta}(\rho) \cdot a) \tilde{\delta}(\rho)$. So $a$ is invariant under all these monodromy transformations iff $\tilde{\delta}(\rho) \cdot a=0$ for all $\rho$. This in turn, is according to (3.4) equivalent to: $a \in I$. Notice that the images of these loops in $\pi_{1}(U, \xi)$ generate the latter, so we may restate the preceding by saying that $I$ is the set of classes in $H_{n-1}\left(X_{\xi}\right)$ that are left invariant by the monodromy action of $\pi_{1}(U, \xi)$.

We mention two other properties equivalent to those of (3.5). The proofs are not difficult, but we won't discuss them here (they can be found for instance in the U. of U.-notes).

(4.8) Proposition. Each of the properties of (3.5) is also equivalent to

1. $V_{\mathbb{C}}$ is a simple representation of $\pi_{1}(U, \xi)$ (i.e., $V_{\mathbb{C}}$ has no nonzero proper $\pi_{1}(U, \xi)$-invariant subspace) and is not the one-dimensional trivial representation, or

2. $H_{n-1}\left(X_{\xi} ; \mathbb{C}\right)$ is a semi-simple representation of $\pi_{1}(U, \xi)$ (this means that every $\pi_{1}(U, \xi)$-invariant subspace of $H_{n-1}\left(X_{\xi} ; \mathbb{C}\right)$ has a $\pi_{1}(U, \xi)$-invariant complement).

Remark. One can show that for $d>0$ sufficiently large, in any general Lefschetz pencil of degree $d$-hypersurface sections of $X$ the special case of (4.6) does not occur (see P. Deligne, Lemme 3.3.7 in Exposé XV of [8]). (Perhaps we should point out that a degree $d$-hypersurface section of $X$ becomes a hyperplane section if we embed $X$ in a higher dimensional projective space by means of the $d$-fold Veronese map.) 


\section{LECTURE 5. The Leray spectral sequence of a Lefschetz fibration}

(5.1) We begin with a definition. Let $M$ be a locally contractible space. A local system over $M$ is a sheaf on $M$ of finite dimensional $\mathbb{Q}$-vector spaces which is locally constant. Local systems are closed under taking pull-back and the binary operations $\otimes$ and $\mathcal{H o m}$. In particular, if $\mathcal{F}$ is a local system, then so is its dual $\mathcal{F}^{\vee}:=\mathcal{H o m}\left(\mathcal{F}, \mathbb{Q}_{M}\right)$.

If $\gamma:[0,1] \rightarrow M$ is a path from $p$ to $q$, then $\gamma^{*} \mathcal{F}$ is locally constant and hence constant. This determines an isomorphism

$$
h_{\gamma}: \mathcal{F}_{p} \cong\left(\gamma^{*} \mathcal{F}\right)_{0} \cong H^{0}\left([0,1], \gamma^{*} \mathcal{F}\right) \cong\left(\gamma^{*} \mathcal{F}\right)_{1} \cong \mathcal{F}_{q}
$$

This isomorphism only depends on the homotopy class of $\gamma$, and behaves in the expected manner with respect to composition and inverses of paths. So if $M$ is path connected and $p$ is a base point, then we have a representation of $\pi_{1}(M, p)$ on the stalk $\mathcal{F}_{p}$. Conversely, a representation of $\pi_{1}(M, p)$ on a finite dimensional $\mathbb{Q}$-vector space $F$ determines a local system as follows: let $\tilde{M} \rightarrow M$ be the universal covering relative $p$ with its action of $\pi_{1}(M, p)$ as deck transformations, and let $\pi_{1}(M, p)$ act on $F \times \tilde{M}$ diagonally (we give $F$ the discrete topology). The orbit space is then the geometric realization of a local system on $M$. These two constructions are inverses of each other (up to a natural isomorphism), so that after a choice of base point $p$, giving a local system on $M$ is essentially the same thing as a giving representation of $\pi_{1}(M, p)$ on a finite dimensional $\mathbb{Q}$-vector space.

Examples of local systems arise as follows. Let $f: Z \rightarrow M$ be a map of spaces The $q$-th direct image (of the constant sheaf $\mathbb{Q}_{Z}$ on $Z$ ) is the sheaf associated to the presheaf

$$
U \stackrel{\text { open }}{\subset} M \mapsto H^{q}\left(f^{-1} U ; \mathbb{Q}\right)
$$

One denotes this sheaf by $R^{q} f_{*} \mathbb{Q}_{z}$. Since $M$ is locally contractible, we have that the stalk at $p \in M$ is just

$$
\left(R^{q} f_{*} \mathbb{Q} Z\right)_{p}=\lim _{U \ni p} H^{q}\left(f^{-1} U ; \mathbb{Q}\right) \cong H^{q}\left(Z_{p} ; \mathbb{Q}\right) .
$$


If $f$ happens to be locally trivial, then this sheaf is locally constant, and if moreover the fibres have finite qth Betti number, then we actually have a local system.

(5.2) In the remainder of this lecture, we let $C$ be a compact connected smooth curve and $S \subset C$ a finite subset. We denote $C-S$ by $U$ and name the inclusions $i: S \subset C$ and $j: U \subset C$. We also choose a base point $\xi \in C$ and we put $\pi_{1}:=\pi_{1}(U, \xi)$.

Suppose $\mathcal{F}$ is a local system on $U$. The direct image $j_{*} \mathcal{F}$ restricted to $U$ is $\mathcal{F}$ again. If $s \in S$ and $D$ is small disk in $C$ about $s$, and $\gamma$ is the loop that traverses $\partial D$ once in the positive direction (starting and ending in some $\eta \in \partial D$ ), then we have isomorphisms

$$
j_{*} \mathcal{F}_{s} \stackrel{\cong}{\longleftarrow} H^{0}\left(D, j_{*} \mathcal{F}\right) \stackrel{\cong}{\longrightarrow} H^{0}\left(\partial D, j_{*} \mathcal{F}\right) \stackrel{\cong}{\longrightarrow}\left(\mathcal{F}_{\eta}\right)^{\gamma}
$$

The following lemma gives a relatively concrete interpretation of the cohomology groups of $j_{*} \mathcal{F}$. (Since $C$ has real dimension two, its cohomology groups in degree $>2$ vanish.)

(5.3) Lemma. We have natural isomorphisms

$$
\begin{aligned}
& H^{0}\left(j_{*} \mathcal{F}\right) \cong\left(\mathcal{F}_{\xi}\right)^{\pi_{1}}, \\
& H^{1}\left(j_{*} \mathcal{F}\right) \cong i m\left(H _ { c } ^ { 1 } \left(U, \mathcal{F} \rightarrow H^{1}(U, \mathcal{F}),\right.\right. \\
& H^{2}\left(j_{*} \mathcal{F}\right) \cong\left(\mathcal{F}_{\xi}\right)_{\pi_{1}},
\end{aligned}
$$

where $H_{c}^{k}(U, \mathcal{F})$ denotes cohomology with compact support, and the natural pairings

$$
H^{k}\left(j_{*} \mathcal{F}\right) \times H^{2-k}\left(j_{*} \mathcal{F}^{\vee}\right) \rightarrow H^{2}(C ; \mathbb{Q}) \cong \mathbb{Q}
$$

are perfect $(k=0,1,2)$.

Proof. We have $H^{0}\left(j_{*} \mathcal{F}\right)=H^{0}(U, \mathcal{F})$ and it is not difficult to see that the restriction map $H^{0}(U, \mathcal{F}) \rightarrow \mathcal{F}_{\xi}$ is an isomorphism onto $\left(\mathcal{F}_{\xi}\right)^{\pi_{1}}$. We next invoke a standard exact sequence:

$$
\cdots \rightarrow H^{k-1}\left(S, i^{*} j_{*} \mathcal{F}\right) \rightarrow H_{c}^{k}(U, \mathcal{F}) \rightarrow H^{k}\left(j_{*} \mathcal{F}\right) \rightarrow H^{k}\left(S, i^{*} j_{*} \mathcal{F}\right) \rightarrow \cdots
$$

Since $i^{*} j_{*} \mathcal{F}$ is a sheaf on the discrete set $S$ it has no higher cohomology, and so $H_{c}^{2}(U, \mathcal{F}) \cong H^{2}\left(j_{*} \mathcal{F}\right)$. Poincaré duality for $\mathcal{F}$ implies that

$$
H^{k}\left(U, \mathcal{F}^{\vee}\right) \times H_{c}^{2-k}(U, \mathcal{F}) \rightarrow H_{c}^{2}(U, \mathbb{Q}) \cong \mathbb{Q}
$$

is a perfect pairing. Since $H^{0}\left(U, \mathcal{F}^{\vee}\right) \cong H^{0}\left(j_{*} \mathcal{F}^{\vee}\right)$, it follows that the pairing in the lemma is perfect for $k=0$. Applying these assertions to $\mathcal{F}^{\vee}$ yields the description of $H^{2}\left(j_{*} \mathcal{F}\right)$ plus the assertion that the pairing is perfect for $k=2$. 
It remains to do the case $k=1$. The exact sequence above gives a surjection

$$
H_{c}^{1}(U, \mathcal{F}) \rightarrow H^{1}\left(j_{*} \mathcal{F}\right)
$$

The Leray spectral sequence of $(j, \mathcal{F})$,

$$
E_{2}^{p, q}:=H^{p}\left(C, \mathcal{R}^{q} j_{*} \mathcal{F}\right) \Rightarrow H^{p+q}(U, \mathcal{F})
$$

gives an injection

$$
H^{1}\left(j_{*} \mathcal{F}\right) \rightarrow H^{1}(U, \mathcal{F})
$$

The composite of (2) and (3) is the natural map, and yields the description of $H^{1}\left(j_{*} \mathcal{F}\right)$. Finally, the homomorphism $H_{c}^{1}\left(j_{*} \mathcal{F}\right) \rightarrow H^{1}\left(j_{*} \mathcal{F}\right)$ equals minus its transpose with respect to the Poincaré pairing (1). Elementary linear algebra then implies that the Poincaré pairing induces a nondegenerate form on the image of this homomorphism.

(5.4) Let now be given a closed irreducible subvariety $Z$ of $C \times \mathbb{P}$ of dimension $n+1 \geq 2$ with the property that the projection $f: Z \rightarrow C$ has all its critical points nondegenerate and with distinct values. We let $S$ be its set of critical values. We abbreviate $R^{k} f_{*} \mathbb{Q}_{z}$ by $\mathcal{R}^{k}$, so that for every $t \in C$ the stalk $\mathcal{R}_{t}^{k}$ can be identified with $H^{k}\left(Z_{t} ; \mathbb{Q}\right)$. Since $f$ is locally trivial over $U$, its restriction $j^{*} \mathcal{R}^{k}$, is a local system.

As for any sheaf on $C$, there is a natural homomorphism

$$
\mathcal{R}^{k} \rightarrow j_{*} j^{*} \mathcal{R}^{k}
$$

Over $U$ this is clearly an isomorphism, so let us explicate this homomorphism on the stalk at some $s \in S$. Choose a small disk $D$ about $s, \eta \in \partial D$, and $\gamma$ as before. Then the homomorphism $\mathcal{R}_{s}^{k} \rightarrow j_{*} j^{*} \mathcal{R}_{s}^{k}$ corresponds to the map

$$
H^{k}\left(Z_{s} ; \mathbb{Q}\right) \cong H^{k}\left(Z_{D} ; \mathbb{Q}\right) \rightarrow H^{k}\left(Z_{\eta} ; \mathbb{Q}\right)^{\gamma}
$$

We encountered this map in (4.6); we showed there that it is an isomorphism for $k \neq n+1$, and that for $k=n+1$ it is either an isomorphism (the usual case) or a surjection with one-demensional kernel (the special case). We conclude:

(5.5) Lemma. The natural homomorphism $\mathcal{R}^{k} \rightarrow j_{*} j^{*} \mathcal{R}^{k}$ is an isomorphism for $k \neq n+1$, and for $k=n+1$ it is surjective with kernel a (skyscraper) sheaf $\mathcal{K}$ whose support is a subset of $S$.

Notice that $\mathcal{R}^{k} \rightarrow j_{*} j^{*} \mathcal{R}^{k}$ induces an isomorphism on cohomology in positve degree. The following theorem is essentially due to Deligne and Grothendieck (Thms. 5.6 and 5.6 .8 of Exposé XVIII in [8]). 
(5.6) Theorem. If the hard Lefschet $z$ theorem holds for the smooth fiber $Z_{\xi}$ of $f$, (relative its embedding in $\mathbb{P}$ ), then the rational Leray spectral sequence

$$
E_{2}^{p, q}:=H^{p}\left(C, \mathcal{R}^{q}\right) \Rightarrow H^{p+q}(Z ; \mathbb{Q})
$$

of $f$ (with rational cofficients) degenerates and the natural map $\mathcal{R}^{n+1} \rightarrow j_{*} j^{*} \mathcal{R}^{n+1}$ has a canonical section.

In somewhat more concrete terms, this means the following: the rational cohomology of $Z$ comes with a natural filtration, the Leray filtration:

$$
0 \subset F_{f}^{2} H^{\bullet}(Z ; \mathbb{Q}) \subset F_{f}^{1} H^{\bullet}(Z ; \mathbb{Q}) \subset F_{f}^{0} H^{\bullet}(Z ; \mathbb{Q})=H^{\bullet}(Z ; \mathbb{Q})
$$

and the theorem says that we have natural isomorphisms

$$
\operatorname{Gr}_{F_{f}}^{k} H^{\bullet}(Z ; \mathbb{Q}) \cong H^{k}\left(C, \mathcal{R}^{\bullet-k}\right), \quad k=0,1,2 .
$$

The subspace $F_{f}^{2} H^{k}(Z ; \mathbb{Q})$ of $H^{k}(Z ; \mathbb{Q})$ can be made explicit: the composite

$$
\begin{aligned}
H^{k}(Z ; \mathbb{Q}) & \supset F_{f}^{2} H^{k}(Z ; \mathbb{Q}) \\
& \cong H^{2}\left(C, \mathcal{R}^{k-2}\right) \cong H^{2}\left(C, j_{*} j^{*} \mathcal{R}^{k-2}\right) \cong H^{k-2}\left(Z_{\xi} ; \mathbb{Q}\right)_{\pi_{1}} \leftarrow H^{2}\left(Z_{\xi} ; \mathbb{Q}\right)
\end{aligned}
$$

is just the Gysin homomorphism of $Z_{\xi} \subset Z$, so that $F_{f}^{2} H^{k}(Z ; \mathbb{Q})$ is the image of this Gysin homomorphism. Similarly, the composite

$$
\begin{aligned}
H^{k}(Z ; \mathbb{Q}) & \rightarrow \operatorname{Gr}_{F_{f}}^{0} H^{k}(Z ; \mathbb{Q}) \\
& \cong H^{0}\left(C, \mathcal{R}^{k}\right) \rightarrow H^{0}\left(C, j_{*} j^{*} \mathcal{R}^{k}\right) \cong H^{0}\left(Z_{\xi} ; \mathbb{Q}\right)^{\pi_{1}} \subset H^{k}\left(Z_{\xi} ; \mathbb{Q}\right)
\end{aligned}
$$

is given by restriction.

Proof of the theorem. Since $E_{2}^{p, q}=0$ unless $p=0,1,2$, we only need to show that

$$
d_{2}: H^{0}\left(\mathcal{R}^{q}\right)=E_{2}^{0, q} \rightarrow E_{2}^{2, q-1}=H^{2}\left(\mathcal{R}^{q-1}\right)
$$

is the trivial map $(q=1,2, \ldots)$.

Let $\eta_{Z} \in H^{2}(Z)$ be the pull-back of the hyperplane class of $\mathbb{P}$. Taking the cup product with this class defines a sheaf-homomorphism

$$
L: \mathcal{R}^{k} \rightarrow \mathcal{R}^{k+2} .
$$

Since $L$ is already defined on the cochain level (for the cup product is), it will commute with the differentials of the spectral sequence. By assumption, $L^{k}$ : $\mathcal{R}^{n-k} \rightarrow \mathcal{R}^{n+k}$ is an isomorphism at $\xi(k \geq 0)$. Since this homomorphism is locally constant on $U$, this is in fact true over $U$. So if we introduce the primitive sheaves

$$
\mathcal{P}^{n-k}:=\operatorname{ker}\left(L^{k+1}: j^{*} \mathcal{R}^{n-k} \rightarrow j^{*} \mathcal{R}^{n+k+2}\right),
$$


we get a Lefschetz decomposition

$$
j^{*} \mathcal{R} \bullet \bigoplus_{k \geq 0} \mathbb{Q}[L] /\left(L^{k+1}\right) \otimes \mathcal{P}^{n-k}
$$

This gives us the natural section of $\mathcal{R}^{n+1} \rightarrow j_{*} j^{*} \mathcal{R}^{n+1}$, namely

$$
j_{*} j^{*} \mathcal{R}^{n+1} \stackrel{L^{-1}}{\cong} j_{*} j^{*} \mathcal{R}^{n-1} \cong \mathcal{R}^{n-1} \stackrel{L}{\rightarrow} \mathcal{R}^{n+1}
$$

It also follows that $d_{2}^{0, n-k}: H^{0}\left(\mathcal{R}^{n-k}\right) \rightarrow H^{2}\left(\mathcal{R}^{n-k-1}\right)$ kills the subspace $H^{0}\left(\mathcal{P}^{n-k}\right)$ $\left.\subset H^{0}\left(j_{*} j^{*} \mathcal{R}^{n-k}\right)=H^{0}\left(\mathcal{R}^{n-k}\right)\right)$, for clearly $L^{k+1} d_{2}^{0, n-k}=d_{2}^{0, n-k} L^{k+1}$ does so and

$$
L^{k+1}: H^{2}\left(\mathcal{R}^{n-k-1}\right) \cong H^{2}\left(j_{*} j^{*} \mathcal{R}^{n-k-1}\right) \stackrel{L^{k+1}}{\cong} H^{2}\left(j_{*} j^{*} \mathcal{R}^{n+k+1}\right) \cong H^{2}\left(\mathcal{R}^{n+k+1}\right) .
$$

If view of the Lefschetz decomposition $(*)$ and the fact that $d_{2}$ and $L$ commute, it follows that $d_{2}$ is zero except possibly

$$
d_{2}^{0, n+1}: H^{0}\left(\mathcal{R}^{n+1}\right)=E_{2}^{0, n+1} \rightarrow E_{2}^{1, n-1}=H^{2}\left(\mathcal{R}^{n}\right) \cong H^{2}\left(j_{*} j^{*} \mathcal{R}^{n}\right) .
$$

We deal with this case by invoking Poincaré duality on both $Z$ and the smooth fibers of $f$. The $k$ th Betti number of $Z$ is given by $\operatorname{dim} E_{\infty}^{0, k}+\operatorname{dim} E_{\infty}^{1, k-1}+\operatorname{dim} E_{\infty}^{2, k-2}$. If $(p, q) \neq(0, n+1),(2, n-1)$, then $\operatorname{dim} E_{\infty}^{p, q}=\operatorname{dim} E_{2}^{p, q}=\operatorname{dim} H^{p}\left(j_{*} j^{*} \mathcal{R}^{q}\right)$, whereas $\operatorname{dim} E_{\infty}^{2, n-1}=\operatorname{coker} d_{2}^{0, n+1}$. By Poincaré duality the $n$th and $(n+2)$ nd Betti number of $Z$ are equal. Writing this out gives

$$
\begin{aligned}
\operatorname{dim} H^{0}\left(j_{*} j^{*} \mathcal{R}^{n}\right) & +\operatorname{dim} H^{1}\left(j_{*} j^{*} \mathcal{R}^{n-1}\right)+\operatorname{dim} H^{2}\left(j_{*} j^{*} \mathcal{R}^{n-2}\right) \\
& =\operatorname{dim} H^{0}\left(j_{*} j^{*} \mathcal{R}^{n+2}\right)+\operatorname{dim} H^{1}\left(\left(j_{*} j^{*} \mathcal{R}^{n+1}\right)+\operatorname{dim} \operatorname{coker} d_{2}^{0, n+1} .\right.
\end{aligned}
$$

Poincare duality on the smooth fibers of $f$ implies that $j^{*} \mathcal{R}^{n+k}$ can be identified with the dual of $j^{*} \mathcal{R}^{n-k}$, so that by (5.5) the spaces $H^{0}\left(j_{*} j^{*} \mathcal{R}^{n+k}\right)$ and $H^{2}\left(j_{*} j^{*} \mathcal{R}^{n-k}\right)$ can be regarded as each others dual. In particular, they have the same dimension. Since $L: j^{*} \mathcal{R}^{n-1} \rightarrow j^{*} \mathcal{R}^{n+1}$ is an isomorphism, we also have $\operatorname{dim} H^{1}\left(j_{*} j^{*} \mathcal{R}^{n-1}\right)=\operatorname{dim} H^{1}\left(j_{*} j^{*} \mathcal{R}^{n+1}\right)$. If we feed this in the displayed equality, we find that $\operatorname{dim} \operatorname{coker} d_{2}^{0, n+1}=\operatorname{dim} H^{2}\left(j_{*} j^{*} \mathcal{R}^{n}\right)$. Hence $d_{2}^{0, n+1}$ is the zero map.

It follows from previous theorem that $\operatorname{Gr}_{F_{f}}^{0} H^{n+1}(Z ; \mathbb{Q}) \cong H^{0}\left(\mathcal{R}^{n+1}\right)$ is canonically isomorphic to the direct sum of $H^{0}(\mathcal{K})$ and $H^{O}\left(j_{*} j^{*} \mathcal{R}^{n+1}\right)$ so that there is a natural surjection $\operatorname{Gr}_{F_{f}}^{0} H^{n+1}(Z ; \mathbb{Q}) \rightarrow H^{0}(\mathcal{K})$. Compose this surjection with the evident projection

$$
H^{\bullet}(Z ; \mathbb{Q}) \rightarrow H^{n+1}(Z ; \mathbb{Q}) \rightarrow \operatorname{Gr}_{F_{f}}^{0} H^{n+1}(Z ; \mathbb{Q})
$$


and denote the kernel by $H^{\bullet}(Z ; \mathbb{Q})$ (so ' $H^{k}(Z ; \mathbb{Q})=H^{k}(Z ; \mathbb{Q})$ if $k \neq n+1$ ). This subspace is invariant under the operator $L$. Let $F_{f}^{k \prime} H^{\bullet}(Z ; \mathbb{Q}):=F_{f}^{k} H^{\bullet}(Z ; \mathbb{Q}) \cap$ $' H \bullet(Z ; \mathbb{Q})$. Then for all $k$ and $m$ we have

$$
G r_{F_{f}}^{k} H^{m}(Z ; \mathbb{Q}) \cong H^{k}\left(j_{*} j^{*} \mathcal{R}^{m-k}\right) .
$$

(5.7) Proposition. The subspace ' $H^{\bullet}(Z ; \mathbb{Q})$ of $H^{\bullet}(Z ; \mathbb{Q})$ is nondegenerate with respect to Poincaré duality, so that its perp (which is a subspace of $H^{n+1}(Z ; \mathbb{Q})$ ) projects isomorphically onto $H^{0}(\mathcal{K})$. Moreover, the filtration $F_{f}^{{ }^{\prime}} H^{\bullet}(Z ; \mathbb{Q})$ is selfdual, in the sense that $F_{f}^{1 \prime} H^{\bullet}(Z ; \mathbb{Q})$ and $F_{f}^{2 \prime} H^{\bullet}(Z ; \mathbb{Q})$ are each others annihilator.

Proof. The Leray filtration on $H^{\bullet}(Z ; \mathbb{Q})$ respects the cup product in the sense that $F_{f}^{k} \cup F_{f}^{l} \subset F_{f}^{k+l}$. So $F_{f}^{1}$ and $F_{f}^{2}$ are perpendicular to each other with respect to Poincaré duality. This implies that Poincaré duality induces pairings

$$
G r_{F_{f}}^{k} H^{m}(Z ; \mathbb{Q}) \times G r_{F_{f}}^{2-k \prime} H^{2 n+2-m}(Z ; \mathbb{Q}) \rightarrow \mathbb{Q}
$$

We must show that these are perfect. But this follows from the fact that this is just the Poincaré duality map

$$
H^{k}\left(j_{*} j^{*} \mathcal{R}^{m}\right) \times H^{2-k}\left(j_{*} j^{*} \mathcal{R}^{2 n-m}\right) \rightarrow \mathbb{Q}
$$

of lemma (5.5).

(5.8) At this point we need the basic results of Hodge theory. We shall give a very brief review; good surveys (and much more) can be found in [11].

Giving a Hodge structure of weight $w \in \mathbb{Z}$ on a finite dimensional $\mathbb{Q}$-vector space $H$ is simply giving a decomposition of its complexificion: $H_{\mathbb{C}}=\oplus_{p+q=w} H^{p, q}$ with the property that $\overline{H^{p, q}}=H^{q, p}$. (Here $p$ and $q$ run over the integers.) This is equivalent to giving a descending filtration $F^{\bullet}=\cdots F^{p} \supset F^{p+1} \cdots$ of $H_{\mathbb{C}}$ which begins with $H$ and ends with $\{0\}$ such that $F^{p} \oplus \bar{F}^{w+1-p} \rightarrow H_{\mathbb{C}}$ is an isomorphism for all $p$; we call this the Hodge filtration of the Hodge structure. (One passes from one set of data to the other by means of the relations $F^{p}=\oplus_{p^{\prime}} \geq p H^{p^{\prime}}, w-p^{\prime}$ and $H^{p, w-p}=F^{p} \cap \bar{F}^{w-p}$.) There is an evident notion of a morphism of Hodge structures $\phi: H \rightarrow ' H$ : we want that $\phi$ maps $H^{p, q}$ to ' $H^{p, q}$; this is equivalent to the apparently weaker condition that $\phi\left(F^{p}\right) \subset{ }^{\prime} F^{p}$ for all $p$, provided that the two Hodge structures have the same weight. There is also the more general notion of a morphism of bidegree $(r, r)$, whose definition you will guess. Practically all the familiar operations with vector spaces have their analogue for Hodge structures, for the category of Hodge structures is abelian and comes with the binary operations Hom and $\otimes$.

A very simple Hodge structure of dimension one is the one-dimensional rational vector space $2 \pi \sqrt{-1} \mathbb{Q}$ whose complexification $\mathbb{C}$ has been given bidegree $(-1,-1)$. This is called the Tate Hodge structure and is denoted $\mathbb{Q}(1)$. Note that complex conjugation acts on it as minus the identity. More generally, $\mathbb{Q}(k), k \in \mathbb{Z}$, is the vector space $(2 \pi \sqrt{-1})^{k} \mathbb{Q}$ with bidegree $(-k,-k)$, so that $\mathbb{Q}(k) \otimes \mathbb{Q}(l) \cong \mathbb{Q}(k+l)$. 
If we twist a weight $m$-Hodge structure $H$ with $\mathbb{Q}(k): H(k):=H \otimes \mathbb{Q}(k)$, then the result is a weight $(m-k)$-Hodge structure; the underlying complex space looks the same, and is the same if we are prepared to fix a square root of -1 . But this one often wants to avoid, and for this reason Tate Hodge structures help us to do the bookkeeping of weights and the action of $\operatorname{Gal}(\mathbb{C} \mid \mathbb{R})$.

Given a Hodge structure of weight $w H$, then the Weil operator $J: H_{\mathbb{C}} \rightarrow H_{\mathbb{C}}$ is defined as multiplication by $\sqrt{-1}^{p-q}$ on $H^{p, q}$. This operator commutes with complex conjugation, so is real. A polarization of $H$ is a morphism of Hodge structures $\psi: H \otimes H \rightarrow \mathbb{Q}(-w)$ such that

1. $\psi$ is $(-)^{w}$-symmetric and

2. $(\alpha, \beta) \mapsto \psi(\alpha, J \bar{\beta})$ is a positive Hermitean form.

The basic results due to Hodge assert among other things the following: if $X$ is a projective manifold (of dimension $n$, say), then $H^{w}(X ; \mathbb{Q}$ ) carries a functorial Hodge structure of weight $w$. The operator $L=\eta_{X} \cup$ of lecture (3) is actually a morphism of Hodge structures $H^{w}(X) \rightarrow H^{w+2}(X)(1)$. In particular, the cohomology group of top degree $H^{2 n}(X ; \mathbb{Q})$ is canonically isomorphic to $\mathbb{Q}(-n)$. If one defines $P_{n-k}(X)$ and $\Psi$ as in that lecture - one does not need the hard Lefschetz theorem to make these definitions - then $\psi:=(2 \pi \sqrt{-1})^{w} \Psi$ is a polarization up to sign. This, of course, implies the nondegeneracy of $\Psi$ and hence the hard Lefschetz theorem.

Griffiths found that if $X$ varies in a holomorphic, $C^{\infty}$ locally trivial family of projective manifolds $X_{t}$, then the Hodge structures on $H^{w}\left(X_{t} ; \mathbb{Q}\right)$ have some remarkable properties concerning their dependence on $t$. This motivated the following definition:

Let $M$ be a complex manifold. A variation of Hodge structure (VHS) of weight $w$ on $M$ is going to be a local system $\mathcal{H}$ on $M$ plus a Hodge structure of weight $w$ on every stalk $\mathcal{H}_{x}$ that varies in a nice way. Here 'nice' means:

1. the Hodge filtration varies holomorphically, i.e., we are given a filtration of

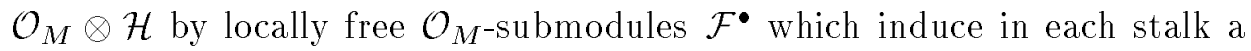
Hodge structure of weight $m$,

2. the Hodge filtration does not vary too much: if $\mathcal{H}$ is trivial over an open connected $U \subset M$, so that $\mathcal{F}^{\bullet} \mid U$ can be thought of as a holomorphic family $\left\{F_{t}^{\bullet}\right\}_{t \in U}$ of filtrations of $H:=H^{0}(U ; \mathcal{H})$, then for any a holomorphic section $s$ of $\mathcal{F}^{p} \mid U$, given as $t \mapsto s(t) \in F_{t}^{p}$, the derivative of $s$ at $t \in U$ maps inside $F_{t}^{p-1}$.

Now if $Z \subset M \times \mathbb{P}$ is a closed complex submanifold such that the projection $f: Z \rightarrow M$ is $C^{\infty}$ locally trivial, then it is a fact that the higher direct images $\mathcal{R}^{w} f_{*} \mathbb{Q}_{Z}$ come naturally with a variation of Hodge structure of weight $m$.

A polarization of a VHS $\mathcal{H}$ of weight $w$ is a homomorphism of local systems $\mathcal{H} \otimes \mathcal{H} \rightarrow \mathbb{Q}_{M}(-w)$ which induces on every stalk a polarization as above.

A key result in the abstract theory of variations of Hodge structure is the following theorem, due to Deligne (cases $k=0,2)$ and Zucker (case $k=1$ ):

(5.9) Theorem. Let $j: U \subset C$ be as before, and let $\mathcal{H}$ be a polarized variation of Hodge structure of weight $w$ over $U$. Then the cohomology group $H^{k}\left(j_{*} \mathcal{H}\right)$ carries a canonical polarized Hodge structure of weight $m+k$. For $k=0$, the polarization 
arises from the isomorphism $H^{0}\left(j_{*} \mathcal{H}\right) \cong\left(\mathcal{H}_{\xi}\right)^{\pi_{1}}$ and the polarization on $\mathcal{H}_{\xi}$, for $k=$ 2 the polarization is similarly defined (it comes from the isomorphism $H^{2}\left(j_{*} \mathcal{H}\right) \cong$ $\left.\left(\mathcal{H}_{\xi}\right)_{\pi_{1}}(-1)\right)$ and for $k=1$ it is given by $H^{1}\left(j_{*} \mathcal{H}\right) \otimes H^{1}\left(j_{*} \mathcal{H}\right) \rightarrow H^{2}\left(C, \mathbb{Q}_{C}(-w)\right) \cong$ $\mathbb{Q}(-w-1)$.

This theorem implies that for every $t \in U, \mathcal{H}_{t}^{\pi_{1}}$ is a Hodge substructure of $\mathcal{H}_{t}$, is constant in $t$ (as a Hodge structure), and is nondegenerate relative the polarization. In view of property of (4) of (3.5) and the interpretation (4.7), we could regard this as an abstract version of the hard Lefschetz theorem.

We will also need the more general notion of mixed Hodge structure. A mixed Hodge structure on a finite dimensional $\mathbb{Q}$-vector space $H$ consists of two filtrations: one increasing filtration $W$. on $H$ which begins with $\{0\}$ and ends with $H$ (the weight filtration) and a decreasing filtration $F^{\bullet}$ of $H_{\mathbb{C}}$ (the Hodge filtration) which induces on every quotient $W_{m} / W_{m-1}$ a Hodge structure $F^{\bullet}\left(W_{m} / W_{m-1}\right)$ of weight $m$ (here $F^{p}\left(W_{m} / W_{m-1}\right)$ is the image of $\left.F^{p} \cap W_{m, \mathbb{C}}\right)$. Given mixed Hodge structures $\left(H, W_{\bullet}, F^{\bullet}\right)$ and $\left(H,{ }^{\prime} W_{\bullet},{ }^{\prime} F^{\bullet}\right)$, then a morphism between them is simply a homomorphism $\phi: H \rightarrow^{\prime} H$ which sends $W_{m}$ to ${ }^{\prime} W_{m}$ and $F^{p}$ to ${ }^{\prime} F^{p}$. It is not-so-trivial fact from linear algebra that this defines an abelian category.

The raison d'être of this definition is the theorem of Deligne which asserts that for every complex-algebraic variety $X$ of finite type, $H^{k}(X ; \mathbb{Q})$ comes with a functorial mixed Hodge structure having $W_{-1}=0$ and $W_{2 k}=H^{k}(X ; \mathbb{Q})$; if $X$ is smooth resp. compact, then we even have $W_{k-1} H^{k}(X ; \mathbb{Q})=0$ resp. $W_{k}=$ $H^{k}(X ; \mathbb{Q})$. He also proved such results for local cohomology groups, as long as they can be defined algebraically.

(5.10) We return to our general Lefschetz pencil $f: \tilde{X} \rightarrow L$, where $X$ has dimension $n \geq 2$. We wish to determine the implications of theorem (5.6) and (5.7) for $H^{\bullet}(X ; \mathbb{Q})$. We first show that $H^{\bullet}(X ; \mathbb{Q})$ is a direct summand of $H^{\bullet}(\tilde{X} ; \mathbb{Q})$.

(5.11) Lemma. We a have a natural isomorphism

$$
H^{\bullet}(\tilde{X} ; \mathbb{Q}) \cong H^{\bullet}(X ; \mathbb{Q}) \perp H^{\bullet-2}(Y ; \mathbb{Q})(-1)
$$

of duality spaces. (The Tate twist $(-1)$ changes the sign of Poincaré pairing of $H^{\bullet}(Y ; \mathbb{Q})$.)

Proof. The projection $\pi:(\tilde{X}, \tilde{Y}) \rightarrow(X, Y)$ induces a morphism of long exact cohomology sequences. This morphism induces an isomorphism on the relative cohomology groups (for $\pi$ is a relative homeomorphism), whereas the induced map $\pi^{*}: H^{k}(Y) \rightarrow H^{k}(\tilde{Y})=H^{k}(Y \times L)$ is injective with cokernel isomorphic to $H^{k-2}(Y)$ (by the Künneth formula). This gives us the a short exact sequence

$$
0 \rightarrow H^{\bullet}(X ; \mathbb{Q}) \stackrel{\pi^{*}}{\rightarrow} H^{\bullet}(\tilde{X} ; \mathbb{Q}) \rightarrow H^{k-2}(Y ; \mathbb{Q}) \rightarrow O
$$

The first map preserves the intersection form and admits $\pi_{\text {! }}$ as a perpendicular section. Using the fact that the normal bundle of $\tilde{Y}=Y \times L$ is the pull-back of $\mathcal{O}_{L}(-1)$, one may complete the proof. 
Let $V(Y)$ be the kernel of the Gysin map $H^{n-2}(Y ; \mathbb{Q}) \rightarrow H^{n}\left(X_{\xi} ; \mathbb{Q}\right)$. Using Poincare duality on $Y$ and $X_{\xi}$, we see that this space can be identified with the kernel of $H_{n-2}(Y ; \mathbb{Q}) \rightarrow H_{n-2}\left(X_{\xi} ; \mathbb{Q}\right)$, that is with the vanishing homology of the pair $\left(X_{\xi}, Y\right)$. (This explains the notation.)

(5.12) Proposition. Suppose that $X$ and its transversal linear sections satisfy the hard Lefschet $z$ theorem. Then we have a natural isomorphism of duality spaces

$$
H^{1}\left(\mathcal{R}^{n-1} f_{*} \mathbb{Q}_{\tilde{X}}\right) \perp H^{0}(\mathcal{K}) \cong P^{n}(X) \perp V(Y)(-1) .
$$

Proof. It follows from the discussion following the statement of (5.6) and (5.7), that the left-hand side is the perp of the image of $i_{\xi !}: H^{n-2}\left(X_{\xi} ; \mathbb{Q}\right) \rightarrow H^{n}(\tilde{X})$ in the kernel of $i_{\xi}^{*}: H^{n}(\tilde{X}) \rightarrow H^{n}\left(X_{\xi} ; \mathbb{Q}\right)$ where $i_{\xi}: X_{\xi} \subset \tilde{X}$. We decompose $H^{n}(\tilde{X} ; \mathbb{Q})$ as

$$
\begin{aligned}
H^{n}(\tilde{X} ; \mathbb{Q}) & =H^{n}(X ; \mathbb{Q}) \perp H^{n-2}(Y ; \mathbb{Q})(-1) \\
& =P^{n}(X) \perp H^{n-2}\left(X_{\xi} ; \mathbb{Q}\right)(-1) \perp H^{n-2}\left(X_{\xi} ; \mathbb{Q}\right)(-1) \perp V(Y)(-1) .
\end{aligned}
$$

One verifies that $i_{\xi !}$ corresponds to the diagonal embedding on the middle two summands, whereas $i_{\xi}^{*}$ corresponds to taking the difference of the components of the middle summands followed by the isomorphism $H^{n-2}\left(X_{\xi} ; \mathbb{Q}\right)(-1) \cong H^{n}\left(X_{\xi} ; \mathbb{Q}\right)$. The proposition follows from this.

Remember that with the notation of lecture (3),

$$
H^{0}(\mathcal{K})=\oplus_{\rho} \operatorname{ker}\left(H^{n}\left(X_{\xi(\rho)}\right) \rightarrow H^{n}\left(X_{\eta(\rho)}\right)\right)
$$

So in a sense the left hand side of (5.12) pertains to the cohomology of hyperplane sections of $X$ only. What makes this proposition interesting is that on the other side of this equality appears as a direct summand that part of the cohomology of $X$ that does not appear in its general hyperplane sections, i.e., $P^{n}(X)$.

This observation may serve as a point of departure for an inductive, though rather indirect, construction of Hodge structures on projective manifolds together with a proof of the hard Lefschetz theorem. The idea is this: suppose we have been able to develop (mixed) Hodge theory in dimension $<n$ (including the relative case of families of projective manifolds of $\operatorname{dim}<n$ ). The induction step then requires us to prove the hard Lefschetz theorem for $X$ and to construct (among othere things) an intrinsic Hodge structure on $P^{n}(X)$ which is (up to a factor) polarized by the intersection product. The hard Lefschetz theorem follows from $(5.9)$, as it implies that $H^{n-1}\left(X_{\xi} ; \mathbb{Q}\right)$ is a semi-simple representation of $\pi_{1}$. The last proposition puts the Hodge structure on $P^{n}(X)$. However, one does not know a priori it is intrinsic. This is only an apparent issue because one proceeds in fact in more specific way: from the outset one has attached to any variety a filtered resolution of the constant complex sheaf of which one wishes to show that the resulting filtration on its cohomology yields the Hodge filtration. The presence of the summand $H^{0}(\mathcal{K})$ forces us to develop mixed Hodge theory at the same time, although that could have been avoided in view of remark at the end of lecture (4). 
The reason for discussing this rather roundabout way of developing Hodge theory is that it generalizes well: it is this approach that has been followed by M. Saito to prove that the intersection cohomology groups of complete irreducible varieties (to be discussed in the next lecture) enjoy similar properties as the cohomology of projective manifolds (i.e., that they have a Hodge structure, that the Hard Lefschetz theorem is valid, etc.). It is similar to the proofs of the corresponding results for varieties defined over finite fields that were obtained earlier by Beilinson-BersteinDeligne. The germ of this idea is already present in Grothendieck's discussion of the Hodge conjecture. 


\section{LECTURE 6. Intersection cohomology}

(6.1) Let $X$ be a locally compact Hausdorff space. A stratification $\mathcal{X}$ of $X$ is a partition of $X$ into connected topological manifolds (called strata) such that the closure of each stratum is a union of strata; the pair $(X, \mathcal{X})$ is then called a stratified space. We have $\operatorname{dim} X=\sup _{S \in \mathcal{X}} \operatorname{dim} S$. If $X$ is a complex algebraic (or analytic) variety, then one usually wants the stratification to consist of smooth subvarieties (locally closed for the Zariski topology); if that is the case, we say that the stratification is algebraic (resp. analytic).

We will be only interested in stratified spaces that have a simple local structure. In order to state the relevant property, recall that the open cone over a space $L$ is what one gets if in $L \times[0, \infty)$, the subspace $L \times\{0\}$ is identified to a point; we denote it by $c(L)$. If $L$ is compact Hausdorff, then a stratification of $L$ determines one of the open cone over it.

We say that a stratification $\mathcal{X}$ of $X$ is locally trivial if for every stratum $S \in$ $\mathcal{X}$ there exists a neighborhood $U_{S}$ of $S$ in $X$, a retraction $r_{S}: U_{S} \rightarrow S$, and a compact stratified space $\left(L_{S}, \mathcal{L}_{S}\right)$ with the the property that $r_{S}$ is locally trivial (with local trivializations respecting the strata) such that any fiber (with its induced stratification) is homeomorphic to the open cone over $\left(L_{S}, \mathcal{L}_{S}\right)$. We actually want that the stratification $\mathcal{L}_{S}$ is also locally trivial; since $\operatorname{dim} L<\operatorname{dim} X$, this makes the definition inductive rather than circular.

It is clear that then every $p \in S$ will have a basis of neighborhoods homeomorphic to $\mathbb{R}^{\operatorname{dim} S} \times c\left(L_{S}\right)$; we shall call any such neighborhood a standard neighborhoodof $p$.

The following theorem is due to Whitney.

(6.2) Theorem. Any complex algebraic (resp. analytic) variety $X$ admits an algebraic (resp. analytic) stratification which is locally trivial.

Actually the stratifications produced by Whitney possess other interesting properties as well; one of them is a generalization of the Ehresmann fibration theorem, which is due to Thom: if $f$ is a proper morphism from $X$ to a smooth variety $M$ such that the restriction of $f$ to any stratum is without critical points, then $f$ is topologically locally trivial. We will refer to these stratifications as Whitney stratifications; for a precise definition, see for example [9]. 
The next theorem makes the definition of intersection cohomology possible; the one following it provides the main reason for its interest. Both are due to Goresky-MacPherson, with modifications in statement and proof due to Deligne.

(6.3) Theorem. Let $(X, \mathcal{X})$ be a locally trivial stratified space. Assume that all the open strata all have the same dimension $m$, and let $\mathcal{F}$ be local system defined on their union $U$. Suppose furthermore that all strata have even codimension. Then there exists a complex of injective sheaves of $\mathbb{Q}_{X}$-modules on $X$,

$$
\mathcal{I}^{\bullet}: 0 \rightarrow \mathcal{I}^{0} \rightarrow \mathcal{I}^{1} \rightarrow \mathcal{I}^{2} \rightarrow \cdots
$$

plus a sheaf homomorphism $\alpha: \mathcal{F} \rightarrow \mathcal{I}^{0} \mid U$ such that the following three properties are satisfied:

1. $0 \rightarrow \mathcal{F} \rightarrow \mathcal{I}^{0}\left|U \rightarrow \mathcal{I}^{1}\right| U \rightarrow \mathcal{I}^{2} \mid U \cdots$ is exact, in other words, $\mathcal{I}^{\bullet} \mid U$ is a resolution of $\mathcal{F}$,

2. (a finiteness property): if $V^{\prime} \subset V$ are standard neighborhoods of $p \in S$, $S \in \mathcal{X}$, then the morphism of complexes

$$
\Gamma\left(V, \mathcal{I}^{\bullet}\right) \rightarrow \Gamma\left(V^{\prime}, \mathcal{I}^{\bullet}\right)
$$

induces an isomorphism of cohomology, and

3. in degrees $\geq \frac{1}{2} \operatorname{codim} S$, the homology of $\Gamma(V, \mathcal{I} \bullet)$ is zero, whereas in degree $<\frac{1}{2} \operatorname{codim} S$ it maps isomorphically onto the cohomology of $\Gamma(V \backslash S, \mathcal{I} \bullet)$. Moreover, the homotopy class of $\mathcal{I}^{\bullet}$ only depends on $X$ and $\mathcal{F}$, not on $\mathcal{X}$.

It follows that in this situation the following spaces are all topological invariants of the pair $(X, \mathcal{F})$ : the intersection cohomology of $(X, \mathcal{F})$ :

$$
I H^{\bullet}(X ; \mathcal{F}):=H^{\bullet}\left(\Gamma\left(X, \mathcal{I}^{\bullet}\right)\right)
$$

the intersection cohomology with compact supports of $(X, \mathcal{F})$ :

$$
I H_{c}^{\bullet}(X ; \mathcal{F}):=H^{\bullet}\left(\Gamma_{c}\left(X, \mathcal{I}^{\bullet}\right)\right),
$$

and for a closed subset $Y \subset X$, the relative intersection cohomology of $(X, \mathcal{F})$ :

$$
I H^{\bullet}(X, Y ; \mathcal{F}):=H^{\bullet}\left(\Gamma_{X-Y}\left(X, \mathcal{I}^{\bullet}\right)\right)
$$

(Here we are only taking sections of $\mathcal{I} \bullet$ with support in $X-Y$.)

Examples. 1. If $X=U$, the we can take for $\mathcal{I}^{\bullet}$ an injective resolution of $\mathcal{F}$, and we find that intersection cohomology is the ordinary cohomology of $\mathcal{F}: I H^{\bullet}(X ; \mathcal{F})=$ $H^{\bullet}(X ; \mathcal{F})$.

2. More generally, if $X$ is manifold and $j: U \subset X$ is the complement of a closed codimension two submanifold, then an injective resolution of $j_{*} \mathcal{F}$ has the required properties and we find that $I H^{\bullet}(X ; \mathcal{F})=H^{\bullet}\left(X ; j_{*} \mathcal{F}\right)$. 
(6.4) Theorem. Suppose that in the the situation of (6.3) $U$ is oriented. Then we have a natural (Poincaré) pairing

$$
I H^{k}(X ; \mathcal{F}) \times I H_{c}^{m-k}\left(X ; \mathcal{F}^{\vee}\right) \rightarrow \mathbb{Q}
$$

which is perfect.

So the Poincaré pairing puts the space $I H^{\bullet}(X, Y ; \mathcal{F})$ in duality with the space $I H^{\bullet}\left(X-Y ; \mathcal{F}^{\vee}\right)$.

In view of the second example, the last assertion of lemma (5.3) is a special case of the above theorem.

We list some other useful properties.

1. If $j: V \subset X$ is open, then we have restriction map $j^{*}: I H^{\bullet}(X ; \mathcal{F}) \rightarrow$ $I H^{\bullet}(V ; \mathcal{F})$ and a pushforward $j_{!}: I H_{c}^{\bullet}(V ; \mathcal{F}) \rightarrow I H_{c}^{\bullet}(X ; \mathcal{F})$,

2. If $\pi: E \rightarrow X$ is locally trivial $\mathbb{R}^{d}$-bundle, then $\pi$ and the zero section induces an isomorphism $I H^{\bullet}\left(E ; \pi^{*} \mathcal{F}\right) \cong I H^{\bullet}(X ; \mathcal{F})$.

3. Let us say that a closed subspace $i: Y \subset X$ is transversally embedded of codimension $d$, if it admits a neighborhood in $X$ which retracts onto $Y$ as an $\mathbb{R}^{d}$-bundle. Then properties (1) and (2) can be combined to yield a natural restriction map $i^{*}: I H^{\bullet}(X ; \mathcal{F}) \rightarrow I H^{\bullet}\left(Y ; i^{*} \mathcal{F}\right)$. It fits in long exact sequence

$$
\cdots \rightarrow I H^{k}(X, Y ; \mathcal{F}) \rightarrow I H^{k}(X ; \mathcal{F}) \rightarrow I H^{k}\left(Y ; i^{*} \mathcal{F}\right) \rightarrow I H^{k+1}(X, Y ; \mathcal{F}) \rightarrow \cdots
$$

The map and the sequence dualize under Poincaré duality to give the Gysin homomorphism $i_{1}: I H^{\bullet-d}\left(Y ; i^{*} \mathcal{F}\right) \rightarrow I H^{\bullet}(X ; \mathcal{F})$ and the long exact sequence

$$
\cdots \rightarrow I H^{k-1}(X-Y ; \mathcal{F}) \rightarrow I H^{k-d}\left(Y ; i^{*} \mathcal{F}\right) \rightarrow I H^{k}(X ; \mathcal{F}) \rightarrow I H^{k}(X-Y ; \mathcal{F}) \rightarrow \cdots
$$

If $Z$ is an arbitrary closed subspace of $Y$, there are also long exact sequences for the triple $(X, Y, Z)$

4. We have a Mayer-Vietoris sequence for open subsets of $X$. (More generally, one can set up a Cech spectral sequence for an open covering of $X$ which converges to the intersection cohomology of $(X, \mathcal{F})$.)

5. If $f$ is a continuous map to a space $Y$, then we have defined the higher direct image sheaf $\mathcal{R}^{k} f_{*} \mathcal{I} \bullet, k=0,1, \ldots$, associated to the presheaf

$$
V \stackrel{\text { open }}{\complement} Y \mapsto I H^{k}\left(f^{-1} V ; \mathcal{F}\right)
$$

and there is a Leray spectral sequence

$$
E_{2}^{p, q}:=H^{p}\left(Y, \mathcal{R}^{k} f_{*} \mathcal{I}^{\bullet}\right) \Rightarrow I H^{p+q}(X ; \mathcal{F}) .
$$

6. If $S \in \mathcal{S}$, and $p \in S$, then it follows from property $(6.3 .2)$ that $I H^{k}(V ; \mathcal{F})$ is independent of the choice of standard neighborhood of $V$ of $p$; we shall call this the local $k$ th intersection cohomology group of $(X, \mathcal{F})$ at $p$. 
7. Property (6.3.3) in combination with property (2) allows us to express the local intersection cohomology groups at $p \in S$ in terms of those of the link $L_{S}:$ if $V$ is a standard neighborhood of $p \in S$, then

$$
I H^{k}(V ; \mathcal{F})=\left\{\begin{array}{l}
I H^{k}(V \backslash S ; \mathcal{F})=I H^{k}\left(L_{S} ; \mathcal{F}\right) \quad \text { if } \quad k \leq d-1 \\
0 \quad \text { if } k \geq d
\end{array}\right.
$$

Applying this to $\mathcal{F}^{\vee}$ and dualizing gives

$$
I H_{c}^{k}(V ; \mathcal{F})=\left\{\begin{array}{l}
0 \quad \text { if } k \leq m-d \\
I H^{(k-m-1+d)+d}\left(L_{S} ; \mathcal{F}\right) \quad \text { if } \quad k \geq m+1-d .
\end{array}\right.
$$

So the first half of the intersection cohomology of $L_{S}$ appears as intersection cohomology of $V$ and the second half appears as intersection cohomology of $V$ with compact supports.

We say that the stratified space $(X, \mathcal{X})$ is of finite type if there exists a compact $K \subset X$ such that $X-K$ is a s a stratified space homeomorphic to the product of a compact stratified space and an open interval. An algebraic Whitney stratification of a quasi-projective variety is always of finite type, but an analytic set need not admit such a stratification (take a countable discrete subset of $\mathbb{C}$ ).

We will sometimes use the notation $I H^{\bullet}(X ; \mathcal{F})$ in a case where $\mathcal{X}$ some strata have odd codimension; we will only do this in case the strata of odd codimension make up a 'boundary' of $X$ : their union $\dot{X}$ should be a closed subspace of $X$ of codimension one and have a neighborhood in $X$ homeomorphic to $X \times[0, \infty)$ as stratified spaces (with the given stratification on $\dot{X}$ and $[0, \infty)$ stratified by $\{0\}$ and $(0, \infty))$. We then let $I H^{\bullet}(X):=I H^{\bullet}(X-\dot{X})$. Notice that if $X$ is compact, then $X-\dot{X}$ is of finite type as a stratified space.

We can now state the weak Lefschetz theorem for intersection cohomology.

(6.5) Weak Lefschetz theorem WL(n). Let $X$ a closed analytic subset of an open convex subset $\Omega$ of a complex affine space which is of pure dimension $n$. Let $\mathcal{X}$ be Whitney stratification of $X$ of finite type and let $\mathcal{F}$ be a local system defined on the union of its open strata. Then $I H^{k}(X ; \mathcal{F})=0$ for $k>n$.

(6.6) Corollary. Let $X \subset \mathbb{P}$ be a projective variety of pure dimension $n, \mathcal{F}$ a local system on a Zariski open-dense subset of $X$, and $H \subset \mathbb{P}$ a hyperplane. Then $I H^{k}(X, X \cap H ; \mathcal{F})=0$ for $k>n$. If moreover $H$ is transversal to a Whitney stratification of $X$, then $i: X \cap H \subset X$ induces a restriction map $i^{*}: I H^{k}(Y ; \mathcal{F}) \rightarrow$ $I H^{k}(X ; \mathcal{F})$ which is an isomorphism for $k \leq n-2$ and is injective for $k=n-1$.

Proof. The first statement follows from $W L(n)$ and the fact that

$$
I H^{k}(X, X \cap H ; \mathcal{F})=I H_{c}^{k}(X-X \cap H ; \mathcal{F}) \cong I H_{c}^{k}\left(X-X \cap H ; \mathcal{F}^{\vee}\right)^{*}
$$

and the second from this and the exact sequence of the pair $(X, X \cap H)$. 
The proof of $W L(n)$ will be by induction on $n$. The induction process yields an interesting local companion result. To state it we need a bit of discussion first.

(6.7) Let $X$ be an analytic set at $0 \in \mathbb{C}^{N}$ of pure dimension $n, \mathcal{X}$ a Whitney stratification of $X, \mathcal{F}$ a local system on the union of its open strata and $f: X \rightarrow$ $\mathbb{C}$ an analytic function with $f(0)=0$. We assume that $f$ has in 0 an isolated singularity at 0 in the sense that there is a neigborhood $\Omega$ of $0 \in X$ such that for any stratum $S \in \mathcal{X}, f \mid S \cap(\Omega-\{0\})$ has no singular point. We construct what we shall call a good model for the germ of $f$ at 0 as follows.

The assumptions (and the properties of a Whitney stratification) imply that $X_{0} \cap(\Omega-\{0\})$ is transversally embedded in $X \cap(\Omega-\{0\})$ and that $\mathcal{X}$ induces a Whitney stratification on it. It is a well-known fact that for every smooth analytic subset $T$ of $\Omega$ (locally closed for the Zariski topology), there exists an $\epsilon>0$ such that $(0, \epsilon]$ does not contain a critical value of the restriction of the norm function $|z|$ to $T$. Choose $\epsilon>0$ such that this is true for all strata of both $\mathcal{X}$ and $\mathcal{X} \mid X_{0} \cap(\Omega-\{0\})$ and assume in addition that the closed $\epsilon$-ball $B(\epsilon)$ is contained $\Omega$.

Now for every $S \in \mathcal{X}, \partial B(\epsilon) \cap S$ and $X_{0} \cap S$ are submanifolds of $S$ which meet transversally. So 0 is not a critical value of $f \mid \partial B(\epsilon) \cap S$. Choose $\eta>0$ such the closed $\eta$-disk $D(\eta)$ in $\mathbb{C}$ consists of regular values of $f \mid \partial B(\epsilon) \cap S$ for all $S$. Put

$$
\begin{aligned}
D & :=D(\eta), \\
B & :=B(\epsilon) \cap f^{-1}(D(\eta), \\
\dot{B} & :=\partial B(\epsilon) \cap f^{-1}(D(\eta), \\
\partial B & :=\left(\partial B ( \epsilon ) \cap f ^ { - 1 } ( D ( \eta ) ) \cup \left(B(\epsilon) \cap f^{-1}(\partial D(\eta)) .\right.\right.
\end{aligned}
$$

On all these spaces we induce the stratification $\mathcal{X}$. The fiber $\left(B_{\eta}, \dot{B}_{\eta}\right)$ is a Whitney stratified space with boundary. We will call $f: B \rightarrow D$ a good model of the germ of $f$ at 0 .

It can be shown that $B$ is homeomorphic to the closed cone over its boundary. This implies that

$$
I H^{k}(B ; \mathcal{F})=\left\{\begin{array}{l}
I H^{k}(\partial B ; \mathcal{F}) \quad \text { if } \quad k \leq n-1 \\
0 \text { if } k \geq n
\end{array}\right.
$$

and

$$
I H_{c}^{k}(B ; \mathcal{F})=\left\{\begin{array}{l}
0 \quad \text { if } k \leq n \\
I H^{k-1}\left(L_{S} ; \mathcal{F}\right) \quad \text { if } \quad k \geq n+1
\end{array}\right.
$$

Thom's generalization of the Ehresmann fibration theorem implies that $\dot{B}$ and $B$ are topologically locally trivial (in a stratified sense) over $D$ and $D-\{0\}$ respectively. We therefore have a geometric monodromy $h:\left(B_{\eta}, \dot{B}_{\eta}\right) \rightarrow\left(B_{\eta}, \dot{B}_{\eta}\right)$ which is the identity on $\dot{B}_{\eta}$. We can even take it to be the identity on a neighborhood of $\dot{B}_{\eta}$ and then it is not hard to define a variation homomorphism for intersection cohomology

$$
\operatorname{var}(h)^{k}: I H^{k}\left(B_{\eta} ; \mathcal{F}\right) \rightarrow I H^{k}\left(B_{\eta}, \dot{B}_{\eta} ; \mathcal{F}\right)
$$


using the sheaf complex $\mathcal{I}^{\bullet}$. We will give another definition below which is somewhat more useful for our purpose.

For this let $\partial_{+} B, \partial_{-} B$ and $\partial_{0} B$ be the set of points of $\partial B$ where $\operatorname{Re}(f)$ is $\geq 0$, $\leq 0$ and $=0$ respectively. Define similarly subspaces $\partial_{+} D, \partial_{-} D$ and $\partial_{0} D$ of $\partial D$. Notice that the pair $\left(\partial_{ \pm} B, \partial_{0} B\right)$ is homeomorphic to the product $\left(B_{ \pm \eta}, \dot{B}_{ \pm \eta}\right) \times$ $\left(\partial_{ \pm} D, \partial_{0} D\right)$, under a homeomorphism whose isotopy class is natural. So we have natural identifications

$$
\begin{aligned}
& I H^{k}\left(\partial_{ \pm} B ; \mathcal{F}\right) \cong I H^{k}\left(B_{ \pm \eta} ; \mathcal{F}\right)=I H^{k}\left(B_{ \pm \eta}-\dot{B}_{ \pm \eta} ; \mathcal{F}\right) \\
& I H^{k}\left(\partial_{ \pm} B, \partial_{0} B ; \mathcal{F}\right) \cong I H^{k-1}\left(B_{ \pm \eta}, \dot{B}_{ \pm \eta} ; \mathcal{F}\right)=I H_{c}^{k-1}\left(B_{ \pm \eta}-\dot{B}_{ \pm \eta} ; \mathcal{F}\right)
\end{aligned}
$$

Now consider the exact sequence of the pair $\left(\partial B, \partial_{+} B\right)$. Since we have

$$
I H^{k+1}\left(\partial B, \partial_{+} B ; \mathcal{F}\right) \cong I H^{k+1}\left(\partial_{-} B, \partial_{0} B ; \mathcal{F}\right) \cong I H^{k}\left(B_{-\eta}, \dot{B}_{-\eta} ; \mathcal{F}\right),
$$

the boundary map of this sequence can be identified with a homomorphism

$$
I H^{k}\left(B_{\eta} ; \mathcal{F}\right) \rightarrow I H^{k}\left(B_{-\eta}, \dot{B}_{-\eta} ; \mathcal{F}\right)
$$

This is essentially the variation homomorphism: each semi-circle in $\partial D$ connecting $-\eta$ with $\eta$ gives a homeomorphism of $B_{-\eta}$ onto $B_{\eta}$, and the two coincide on $\dot{B}_{-\eta}$. The above map is induced by the difference of their actions on the intersection cohomology complex. So if we use one of them to identify $I H^{k}\left(B_{\eta} ; \mathcal{F}\right)$ with $I H^{k}\left(B_{-\eta} ; \mathcal{F}\right)$, then we get the variation homorphism. The preceding discussion shows:

(6.8) Lemma. We have a natural long exact sequence

$$
\cdots \rightarrow I H^{k}(\partial B ; \mathcal{F}) \rightarrow I H^{k}\left(B_{\eta} ; \mathcal{F}\right) \stackrel{\operatorname{var}(h)^{k}}{\longrightarrow} I H^{k}\left(B_{\eta}, \dot{B}_{\eta} ; \mathcal{F}\right) \rightarrow I H^{k+1}(\partial B ; \mathcal{F}) \rightarrow \ldots
$$

We are now ready to state the local companion alluded to above

(6.9) Proposition VAR(n). We have a natural isomorphism

$$
I H^{k}\left(B, \partial_{+} B ; \mathcal{F}\right) \cong \operatorname{Image}\left(\operatorname{var}(h)^{k-1}: I H^{k-1}\left(B_{\eta} ; \mathcal{F}\right) \rightarrow I H^{k-1}\left(B_{\eta}, \dot{B}_{\eta} ; \mathcal{F}\right)\right) .
$$

and both members are trivial for $k \neq n$. In particular, the monodromy action on $I H^{k}\left(B_{\eta} ; \mathcal{F}\right)$ is the identity if $k \neq n-1$. Finally,

$$
I H^{k}\left(B_{\eta} ; \mathcal{F}\right)\left\{\begin{array}{l}
=0 \quad \text { for } k \geq n ; \\
\cong I H^{k}(\partial B ; \mathcal{F}) \quad \text { for } k \leq n-2 .
\end{array}\right.
$$

Remark. So the above proposition implies that if $X$ is smooth, then $H^{k}\left(B_{\eta} ; \mathcal{F}\right)=$ $I H^{k}\left(B_{\eta} ; \mathcal{F}\right)$ is trivial unless $k=0, n-1$. This is an old result of Milnor. 
Proof that $W L(n-1) \Rightarrow V A R(n)$. The hypotheses of $W L(n-1)$ are fulfilled by the Whitney stratified space $B_{\eta}-\dot{B}_{\eta}$, and so $I H^{k}\left(B_{\eta} ; \mathcal{F}\right)=0$ for $k \geq n$ and $I H^{k}\left(B_{\eta}, \dot{B}_{\eta} ; \mathcal{F}\right)=0$ for $k \leq n-2$. We identify the variation homomorphism with the boundary map above, and factor this boundary map as

$$
I H^{k}\left(\partial_{+} B ; \mathcal{F}\right) \stackrel{\delta}{\longrightarrow} I H^{k+1}\left(B, \partial_{+} B ; \mathcal{F}\right) \stackrel{\gamma}{\longrightarrow} I H^{k+1}\left(\partial B, \partial_{+} B ; \mathcal{F}\right) .
$$

Either map fits in an exact sequence:

$$
\begin{gathered}
I H^{k}\left(\partial_{+} B ; \mathcal{F}\right) \stackrel{\delta}{\longrightarrow} I H^{k+1}\left(B, \partial_{+} B ; \mathcal{F}\right) \rightarrow I H^{k+1}(B) \\
I H^{k+1}(B, \partial B ; \mathcal{F}) \rightarrow I H^{k+1}\left(B, \partial_{+} B ; \mathcal{F}\right) \stackrel{\gamma}{\longrightarrow} I H^{k+1}\left(\partial B, \partial_{+} B ; \mathcal{F}\right) .
\end{gathered}
$$

So $\delta$ is surjective if $k \geq n-1$ and $\gamma$ is injective for $k \leq n$. The assertions of $V A R(n)$ all follow from this.

Proof that $W L(n-1)+V A R(n) \Rightarrow W L(n)$. The proof will be very similar to the discussion (2.7). But here we must pay attention to the topological type because intersection homology is not a homotopy invariant. We need a technical result which we merely state:

(6.10) Technical lemma. In the sitation of $W L(n)$, there exists a compact convex set $C \subset \Omega$ with smooth boundary $\partial C$ which is transversal to the stratification $\mathcal{X}$, an affine-linear form $f$, and a convex disk $D \subset \mathbb{C}$ with smooth boundary $\partial D$ such that

1. with respect to the stratification induced on $\partial C \cap X, f \mid \partial C \cap X$ has no critical value in $D$,

2. with respect to the stratification induced on int $(C) \cap X, f \mid \operatorname{int}(C) \cap X$ has only finitely many critical points over $D$, and these critical points have distinct values, which are all contained in int $(D)$,

3. if we put $B:=C \cap f^{-1} D \cap X$, then $X-B$ is homeomorphic to $\partial B \times \mathbb{R}$ as stratified spaces.

Let $S \subset \operatorname{int}(D)$ be the finite set of critical values of $f: B \rightarrow D$. If $x_{s}$ is the critical point over $s \in S$, then choose neighborhoods $B(s) \ni x_{s}$ in $X \cap \operatorname{int}(C) \cap f^{-1} D$ and $D(s) \ni s$ in $\operatorname{int}(D)$ such that $f: B(s) \rightarrow D(s)$ is a good model of the germ of $f \mid X$ at $x_{s}$. We also suppose that the disks $D(s)$ are disjoint. Choose a smoothly embedded disk $E \subset D$ with the property that for every $s \in S, E \cap D(s)$ is the right semi-disk $D(s)_{+}$of $D(s)$. Put

$$
B^{\prime}:=B_{E} \cup \bigcup_{s \in S} B(s), \quad \text { and } \quad B^{\prime \prime}:=\operatorname{clos}\left(B_{E} \backslash \bigcup_{s \in S} B(s)\right)
$$

One proves that $X$ is obtained from $B^{\prime}$ by putting an open collar over its boundary. So $I H^{k}(X ; \mathcal{F}) \cong I H^{k}\left(B^{\prime} ; \mathcal{F}\right)$. Similarly, if $\eta \in E-S$, then $B^{\prime \prime}$ is homeomorphic to 
$B_{\eta}$ cross a disk. So $I H^{k}\left(B^{\prime \prime} ; \mathcal{F}\right) \cong I H^{k}\left(B_{\eta} ; \mathcal{F}\right)$. We may apply $W L(n-1)$ to the relative interior of $B_{\eta}$ and find that the latter group is zero for $k \geq n$. Furthermore,

$$
I H^{k}\left(B^{\prime}, B^{\prime \prime} ; \mathcal{F}\right) \cong \bigoplus_{s \in S} I H^{k}\left(B(s), \partial B(s)_{+} ; \mathcal{F}\right)
$$

and according to $V A R(n)$, this group is trivial unless $k=n$. If we feed this in the exact sequence of the pair $\left(B^{\prime}, B^{\prime \prime}\right)$, the assertion $W L(n)$ follows.

We have only a partial analogue of the results mentioned in (4.6). Let $C$ be a smooth curve, $s \in C, Z \subset \mathbb{P} \times C$ a closed subvariety of pure dimension $n$ equipped with a Whitney stratification $\mathcal{Z}$ and $\mathcal{F}$ a local system over the union of open strata of $\mathcal{Z}$. Denote the projection $f: Z \rightarrow C$, and assume that $f$ has exactly one critical point $z$ over $s$. Let $B \ni z_{0}$ be a closed neighborhood of $z_{0}$ in $Z$ such that $f: B \rightarrow f(B):=D$ is a good representative of the germ of $f$ at $z_{0}$, and assume that $D$ is so small that it does not contain any critical value besides $t$.

(6.11) Corollary. The restriction map $I H^{k}\left(X_{D} ; \mathcal{F}\right) \rightarrow I H^{n}\left(X_{\eta} ; \mathcal{F}\right)$ is an isomorphism for $k \neq n-1, n$ and we have an exact sequence

$$
\begin{aligned}
0 \rightarrow I H^{n-1}\left(Z_{D} ; \mathcal{F}\right) & \rightarrow I H^{n-1}\left(Z_{\eta} ; \mathcal{F}\right) \\
& \rightarrow I H^{n}\left(B, B_{\eta} ; \mathcal{F}\right) \rightarrow I H^{n}\left(Z_{D} ; \mathcal{F}\right) \rightarrow I H^{n}\left(Z_{\eta} ; \mathcal{F}\right) \rightarrow 0 .
\end{aligned}
$$

Proof. This follows from $(6.10)$ and the fact that we have an isomorphism

$$
I H^{k}\left(Z_{D}, Z_{\eta}, \mathcal{F}\right) \rightarrow I H^{k}\left(B, B_{\eta} ; \mathcal{F}\right)
$$

The first statement of this corollary can be restated as follows: the sheaf on $C$ associated to the presheaf

$$
(V \stackrel{\text { open }}{\complement} C) \mapsto I H^{k}\left(f^{-1} V ; \mathcal{F}\right)
$$

is constant on $D$ if $k \neq n-1$. In the missing dimension one would like to have an invariant cycle theorem stating that the image of the map $I H^{n-1}\left(Z_{D} ; \mathcal{F}\right) \rightarrow$ $I H^{n-1}\left(Z_{\eta} ; \mathcal{F}\right)$ is just the part that is pointwise fixed under the monodromy transformation. (We proved this in case $Z$ is smooth at $z_{0}$ and $f$ has there a nondegenerate singularity.) This is true in case $\mathcal{F}$ underlies a variation of Hodge structure. However, the proof is difficult, and appears as a byresult of the proof of the hard Lefschetz theorem for intersection cohomology.

Consider the situation of (6.7), where $X \subset \mathbb{P}$ is a projective variety of pure dimension $n, \mathcal{F}$ a local system defined on a smooth Zariski open-dense subset $U$ of $X$ and $H \subset \mathbb{P}$ be a hyperplane transversal with respect to $U$ and a Whitney stratification of $X-U$. The inclusion $i_{H}: X \cap H \subset X$ induces

$$
i^{*}: I H^{n-1}(X ; \mathcal{F}) \rightarrow I H^{n-1}(X \cap H ; \mathcal{F}), \quad i_{!}: I H^{n-1}(X \cap H ; \mathcal{F}) \rightarrow I H^{n+1}(X ; \mathcal{F})
$$


Generalizing terminology for the smooth case, we call the image of the first map the invariant intersection cohomology and the kernel of the second map the vanishing intersection cohomology of $X \cap H$. Then proposition (3.5) and its extension (4.8) hold also for this case. The hard Lefschetz theorem in the present context asserts that these equivalent properties all hold if the local system underlies a polarized VHS; it is due to Morihiko Saito:

(6.12) Theorem (Kähler package for intersection cohomology). Let $X \subset \mathbb{P}$ be $a$ projective variety of pure dimension $n, \mathcal{H}$ a polarized VHS of weight $w$ on a smooth Zariski open-dense subset $U$ of $X$ and $H \subset \mathbb{P}$ be a hyperplane transversal with respect to $U$ and a Whitney stratification of $X-U$. Then

1. I $H^{k}(X ; \mathcal{H})$ carries a Hodge structure of weight $w+k$, which has all the expected functoriality properties: it only depends on $(X, \mathcal{H})$ (so not on the embedding $X \subset \mathbb{P}$ ), is functorial in $\mathcal{H}$, is compatible with the Poincaré pairing in the sense that

$$
I H^{k}(X ; \mathcal{H}) \times I H^{2 n-k}\left(X ; \mathcal{H}^{\vee}\right) \rightarrow \mathbb{Q}(-n)
$$

is a morphism of Hodge structures, and is functorial with respect to restriction to a transversally embedded closed subvariety of pure dimension. In particular, the map $L:=i_{1} i^{*}: I H^{k}(X ; \mathcal{H}) \rightarrow I H^{k+2}(X ; \mathcal{H})(1)$ is a morphism of Hodge structures of weight $w+k$.

2. For $k \geq 0$, the map $L^{k}: I H^{n-k}(X ; \mathcal{H}) \rightarrow I H^{n+k}(X ; \mathcal{H})(k)$ is an isomorphism of Hodge structures, and the resulting map

$$
\psi: I H^{n-k}(X ; \mathcal{H}) \times I H^{n-k}(X ; \mathcal{H}) \rightarrow \mathbb{Q}(k-n), \quad \psi(\alpha, \beta):=L^{k}(\alpha) \cdot \beta,
$$

polarizes the Hodge structure on $I H^{n-k}(X ; \mathcal{H})$.

The proof follows essentially the pattern sketched at the end of lecture 5, but to make the induction run requires us to know that if $X$ varies in an algebraic family, then the Hodge structures on the intersection cohomology groups of the fibres determine a variation of Hodge structure over a Zariski open-dense of the parameter space. Once this has been established, then Hodge theory is developed in relative dimension $n$ far enough so that a weight argument can be used to prove the invariant cycle theorem for relative dimension $n$.

The preceding results admit a powerful generalization to the relative case, the decomposition theorem plus the relative Lefschetz theorem. For the statements, we refer to Saito's overview [14]. 


\section{BIBLIOGRAPHY}

1. A. Borel (ed.), Intersection Cohomology, Progress in Math., Birkhäuser, 1984.

2. A.A. Beilinson, J. Berstein, P. Deligne, Faisceaux Pervers, Astérisque 100 (1982).

3. J. Cheeger, M. Goresky, R. MacPherson, $L^{2}$-Cohomology and intersection homology of singular algebraic varieties, Seminar on Differential Geometry (S.-T. Yau, ed.), Ann. of Math. Studies, vol. 102, Princeton U.P., 1982.

4. P. Deligne, Théorème de Lefschetz et critères de dégénérescence de suites spectrales, Inst. Hautes Etudes Sci. Publ. Math. 35 (1968), 259-126.

5. P. Deligne, Théorie de Hodge I, Congrès Intern. Math. Nice, 1970, pp. 425430; II, Inst. Hautes Études Sci. Publ. Math. 40 (1971), 5-58; III, Inst. Hautes Études Sci. Publ. Math. 44 (1975), 6-77.

6. P. Deligne, Poids dans la Cohomologie des Variétés Algebriques, Intern. Congress Math. Vancouver, 1974, pp. 79-85.

7. P. Deligne, La conjecture de Weil II, Inst. Hautes Études Sci. Publ. Math. 52 (1980), 137-252.

8. P. Deligne and N. Katz (eds.), SGA VII, Lecture Notes in Math., vol. 340, Springer Verlag, 1973.

9. C.G. Gibson et al., Notes on Topological Stability, Lecture Notes in Math., vol. 552, Springer Verlag, 1976.

10. M. Goresky and R. MacPherson, Stratified Morse theory, Ergebnisse der Mathematik, vol. 14, Springer Verlag, 1987.

11. Ph. Griffiths (ed.), Topics in transcendental algbraic geometry, Ann. of Math. Studies, vol. 106, 1984.

12. K. Lamotke, The topology of complex varieties after S. Lefschetz, Topology 20 (1986), $15-51$.

13. S. Lefschetz, l'Analysis Situs et la Géometrie Algebrique, Collection de Monographies sur le théorie des fonctions, Gauthier-Villars et Cie., 1924.

14. M. Saito, Introduction to mixed Hodge modules, Astérisque 179-180 (1987), $145-162$.

15. S. Zucker, Hodge theory with degenerating coefficients: $L_{2}$ cohomology in the Poincaré metric, Ann. Math. 109 (1979), 415-476. 